\title{
Reduction of self-diffusion coefficient in a coarse-grained model of cytoplasm
}

\author{
Tomoshige Miyaguchi ${ }^{*}$ * \\ Department of Mathematics, Naruto University of Education, Tokushima 772-8502, Japan
}

(Received 24 September 2019; accepted 6 February 2020; published 9 March 2020)

\begin{abstract}
Theoretical predictions for polydisperse hard-sphere suspensions with and without hydrodynamic interaction are applied to a coarse-grained model of bacterial cytoplasm, which consists of 15 species of spherical particles. Short-time and long-time self-diffusion coefficients of each species are obtained to the first order in concentration. It is shown that the hydrodynamic interaction leads to a large reduction of diffusivity for small particles such as green fluorescent proteins. Moreover, a heuristic modification of the above theory to make it valid at higher concentrations is presented.
\end{abstract}

DOI: 10.1103/PhysRevResearch.2.013279

\section{INTRODUCTION}

There is considerable interest in macromolecular diffusion inside cells [1-5] because it plays important roles in various intracellular processes such as chemical reactions and information transmission [6]. Indeed, it is found that the intracellular diffusion of macromolecules is remarkably slow compared with diffusion at infinite dilution. For example, the diffusion coefficient of green fluorescent proteins (GFPs) in cytoplasm is about 10 times less than that at infinite dilution [7-9].

To clarify the origin of such a drastic reduction of the diffusivity, coarse-grained models of bacterial cytoplasm have been studied intensively [10-13]. In these coarse-grained models, macromolecules are represented by Brownian spheres with various sizes, and thus they are polydisperse colloidal suspension models. For example, in Ref. [11], Ando and Skolnick numerically studied a coarse-grained model and reported that the reduction of diffusivity observed in experiments for the GFPs is not explained only by the excluded volume effect, but hydrodynamic interaction (HI) between the macromolecules plays a crucial role.

Colloidal suspensions have been analytically studied for many decades [14,15], and analytical expressions for the diffusion coefficient $D(\phi)$ as a function of the volume fraction $\phi$ of the suspended particles are available [16-18]. For example, for monodisperse suspensions (identical Brownian hard spheres) without the HI, the diffusion coefficient is given by $D(\phi) \simeq D(0)(1-2 \phi)$ [15]; similarly, for monodisperse suspensions with the $\mathrm{HI}$, it is given by $D(\phi) \simeq D(0)(1-$ $2.10 \phi)[19,20]$. These theoretical predictions are valid at low concentrations (i.e., at small values of $\phi$ ). According to these

\footnotetext{
*tmiyaguchi@naruto-u.ac.jp

Published by the American Physical Society under the terms of the Creative Commons Attribution 4.0 International license. Further distribution of this work must maintain attribution to the author(s) and the published article's title, journal citation, and DOI.
}

predictions, the diffusion coefficient $D(\phi)$ does not change greatly by incorporating the HI.

These analytical results, however, are contrasting to the above-mentioned simulation study of a coarse-grained model of cytoplasm, in which the diffusion coefficient shows a large decrease by incorporating the HI [11]. This apparent contradiction must be caused by the fact that the above theories are for monodisperse suspensions and valid only at low concentrations, whereas real cytoplasm is highly concentrated and polydisperse.

This paper studies the effect of polydispersity on the reduction of diffusivity in a coarse-grained model of cytoplasm. A theoretical prediction for the reduced diffusivity of monodisperse suspensions without the HI is given in Refs. [15,21]; here, this theoretical prediction is generalized to the polydisperse suspensions. In contrast, the reduced diffusivity in polydisperse suspensions with the HI was theoretically studied by Batchelor [16-18]. In the Batchelor's theory, the HI is taken into account through the so-called mobility functions. In this paper, far-field approximations of these mobility functions are obtained by twin multipole expansions [14,22]. Heuristic modifications of these theories are also presented to make them valid at higher concentrations.

These theoretical formulas are then applied to the coarsegrained model of cytoplasm proposed in Ref. [11], which is a polydisperse colloidal suspension model. Colloidal particles in this model are hard-sphere particles and classified into 15 kinds of macromolecules, that include GFP, RNA polymerase, and ribosome. Hereafter, this colloidal suspension model is referred to as the Ando-Skolnick model. In Ref. [11], roles of nonspecific attractive interactions, molecular shapes, the HI, and the excluded volume effect in reduction of diffusion coefficients were intensively studied, and it was found that the HI and the excluded volume effect are sufficient to reproduce the drastic reduction in diffusivity observed in the experiments. Therefore, only the HI and the hard-sphere excluded volume effect are taken into account in the models of this paper.

By comparing the above two theoretical predictions for the Ando-Skolnick model with and without the HI, it is shown that the diffusivity decreases prominently for small particles by incorporating the HI. For example, it is found that the 
TABLE I. Parameters and mobility coefficients with and without the HI for the Ando-Skolnick model. The Ando-Skolnick model (a polydisperse colloidal suspension model) consists of 15 species of particles. The diffusion coefficient at infinite dilution $\tilde{D}_{s}$ and the particle radius $\tilde{a}_{s}$ are related by the Stokes law $\tilde{a}_{s}=k_{B} T / 6 \pi \eta \tilde{D}_{s}(s=1, \ldots, 15) . K_{s}^{\mathrm{I}}$ and $K_{s}^{\mathrm{B}}$ are the mobility coefficients of the species $s$ for the system without the HI, and $K_{s}^{\mathrm{hS}}, K_{s}^{\mathrm{hI}}, K_{s}^{\mathrm{hB}}$, and $K_{s}^{\mathrm{h}}$ for the system with the HI. The results for the monodisperse suspension [15] are also given for a comparison.

\begin{tabular}{|c|c|c|c|c|c|c|c|c|c|c|}
\hline$s$ & Name & $\begin{array}{c}\text { No. of } \\
\text { particles } n_{s}\end{array}$ & $\begin{array}{l}\text { Diffusion const. } \\
\tilde{D}_{s}\left(\mu \mathrm{m}^{2} / s\right)\end{array}$ & $\begin{array}{l}\text { Radius } \\
\tilde{a}_{s}(\mathrm{~nm})\end{array}$ & $K_{s}^{\mathrm{I}}$ & $K_{s}^{\mathrm{B}}$ & $K_{s}^{\mathrm{hS}}$ & $K_{s}^{\mathrm{hI}}$ & $K_{s}^{\mathrm{hB}}$ & $K_{s}^{\mathrm{h}}$ \\
\hline & Monodisperse system & & & & 2.00 & 0.0 & 1.83 & 0.00 & 0.275 & 2.11 \\
\hline 1 & Ribonuclease HI & 57 & 114.0 & 2.15 & 0.93 & 0.0 & 1.16 & 0.00 & 0.397 & 1.56 \\
\hline 2 & GFP & 93 & 102.0 & 2.41 & 1.00 & 0.0 & 1.22 & 0.00 & 0.388 & 1.61 \\
\hline 3 & $\begin{array}{l}\text { Malonyl CoA-acyl } \\
\text { carrier }\end{array}$ & 77 & 95.2 & 2.58 & 1.04 & 0.0 & 1.26 & 0.00 & 0.382 & 1.64 \\
\hline 4 & Initial tRNA & 299 & 88.3 & 2.78 & 1.09 & 0.0 & 1.30 & 0.00 & 0.376 & 1.68 \\
\hline 5 & $\begin{array}{l}\text { Triosephospate } \\
\text { isomerase }\end{array}$ & 103 & 77.3 & 3.17 & 1.20 & 0.0 & 1.38 & 0.00 & 0.363 & 1.74 \\
\hline 6 & Enolase & 75 & 68.2 & 3.60 & 1.32 & 0.0 & 1.45 & 0.00 & 0.350 & 1.80 \\
\hline 7 & $\begin{array}{l}\text { Fructose } 1-6 \\
\text { bisphosphate aldolase }\end{array}$ & 75 & 66.7 & 3.68 & 1.34 & 0.0 & 1.46 & 0.00 & 0.348 & 1.81 \\
\hline 8 & $\begin{array}{l}\text { 6-phosphogluconate } \\
\text { dehydrogenase }\end{array}$ & 26 & 62.6 & 3.92 & 1.41 & 0.0 & 1.49 & 0.00 & 0.341 & 1.84 \\
\hline 9 & $\begin{array}{l}\text { Phosphoenolpyruvate- } \\
\text { protein } \\
\text { Phosphotransferase }\end{array}$ & 28 & 57.7 & 4.25 & 1.52 & 0.0 & 1.54 & 0.00 & 0.332 & 1.87 \\
\hline 10 & $\begin{array}{l}\text { Glyceraldehyde-3-P } \\
\text { dehydrogenase }\end{array}$ & 35 & 56.9 & 4.31 & 1.54 & 0.0 & 1.55 & 0.00 & 0.331 & 1.88 \\
\hline 11 & $\begin{array}{l}\text { Cystathioine } \\
\text { gamma-synthase }\end{array}$ & 5 & 54.0 & 4.54 & 1.61 & 0.0 & 1.58 & 0.00 & 0.325 & 1.90 \\
\hline 12 & $\begin{array}{l}\text { Phosphoglycerate } \\
\text { dehydrogenase }\end{array}$ & 10 & 49.9 & 4.92 & 1.73 & 0.0 & 1.62 & 0.00 & 0.316 & 1.93 \\
\hline 13 & RNA polymerase & 62 & 36.9 & 6.65 & 2.37 & 0.0 & 1.77 & 0.00 & 0.277 & 2.05 \\
\hline 14 & GroEL/ES & 31 & 28.8 & 8.52 & 3.18 & 0.0 & 1.88 & 0.00 & 0.243 & 2.12 \\
\hline \multirow[t]{2}{*}{15} & Ribosome & 24 & 21.3 & 11.52 & 4.74 & 0.0 & 2.00 & 0.00 & 0.199 & 2.20 \\
\hline & Total & 1000 & & & & & & & & \\
\hline
\end{tabular}

diffusion coefficient for the GFP is estimated as $D_{\mathrm{GFP}}(\phi) \simeq$ $D_{\mathrm{GFP}}(0)(1-1.00 \phi)$ for the system without the HI, and by $D_{\mathrm{GFP}}(\phi) \simeq D_{\mathrm{GFP}}(0)(1-1.61 \phi)$ for the system with the HI. Thus, in contrast to the monodisperse suspension mentioned above, the diffusion coefficient of the GFP shows a large decrease by incorporating the HI. Furthermore, for the case without the HI, the theoretical predictions are compared with numerical simulations of the Ando-Skolnick model, and a good agreement is found even for relatively high concentrations. For a numerical scheme, an event-driven Brownian dynamics simulation method is used [23,24].

Although the main focus of this paper is reduction of the self-diffusion coefficient, anomalous subdiffusion [25-27] is also observed in many experiments [1-3] and coarsegrained models of cytoplasms [28-31]. In fact, single-particletracking experiments revealed that relatively large particles with radii greater than $100(\mathrm{~nm})$ often show anomalous subdiffusion in cytoplasms [1-3].

In contrast, for small particles with radii less than $10(\mathrm{~nm})$ (e.g., proteins), it has been still difficult to observe intracellular subdiffusion directly by single-particle-tracking techniques because of the rapid diffusion speed and difficulty in imaging in depth [32]. Nevertheless, several theoretical and numerical studies predict that subdiffusion (if any) may be observed only transiently, and normal diffusion should be recovered at long times [26,29-31]. Consistently, the particles in the Ando-Skolnick model are relatively small with radii between 2 to $12(\mathrm{~nm})$, and exhibit normal diffusion at long times. In this paper, we focus on this long-time normal diffusion.

This paper is organized as follows. In Sec. II, the polydisperse hard-sphere suspension without the HI is theoretically analyzed by means of a steady Smoluchowski equation, and the results are applied to the Ando-Skolnick model. Results of numerical simulations without the $\mathrm{HI}$ are also presented in this section. In Sec. III, the Ando-Skolnick model with the HI is analyzed with the polydisperse suspension theory. Section IV is devoted to a summary and discussion. In Appendix A, the numerical scheme for the Brownian hard spheres is briefly explained, while, in Appendix B, numerical procedures to determine the long-time diffusion coefficient are explained. Finally, Appendix $\mathrm{C}$ is devoted to theoretical derivations of shortand long-time self-diffusion coefficient for the hard-sphere suspension with the HI. The data summarized in Table I are obtained with the far-field approximation up to $O\left(1 / r^{100}\right)$, whereas exact expressions up to $O\left(1 / r^{7}\right)$ are presented in Appendix C. 


\section{WITHOUT HYDRODYNAMIC INTERACTION}

In this section, we study a polydisperse hard-sphere suspension without the HI, and derive a formula for the diffusion coefficient as a function of the volume fraction $\phi$ by using a steady solution of the Smoluchowski equation. The theoretical analysis in this section is a straightforward generalization of that for the identical hard spheres [15,21].

\section{A. Smoluchowski equation}

Suppose that a hard-sphere suspension consists of $N$ Brownian particles and each particle has a diffusion coefficient $D_{i}(i=1, \ldots, N)$ at infinite dilution. The radii of the particles are assumed to be given by the Stokes radii $a_{i}=$ $k_{B} T /\left(6 \pi \eta D_{i}\right)(i=1, \ldots, N)$, where $k_{B}$ is the Boltzmann constant, $T$ is the temperature, and $\eta$ is the viscosity of the water. Moreover, these Brownian particles are assumed to be classified into $S$ species; the particles belonging to the same species are identical and therefore have the same diffusion coefficient and radius. The number of particles in the species $s$ is denoted as $n_{s}$, and a cumulative number of particles $N_{s}$ are defined by $N_{s}:=\sum_{k=1}^{s} n_{k}(s=1, \ldots, S)$. We also use a notation $\tilde{a}_{s}(s=1, \ldots, S)$ to indicate the radius of the species $s$. Moreover, the indices $i, j$, and $l$ are used to indicate the particle indices, whereas $s$ and $k$ are used to indicate the species indices.

The Smoluchowski equation for the interacting Brownian particles is given by [15]

$$
\frac{\partial}{\partial t} P(\boldsymbol{R}, t)=\hat{\mathcal{L}} P(\boldsymbol{R}, t),
$$

where $P(\boldsymbol{R}, t)=P\left(\boldsymbol{r}_{1}, \ldots, \boldsymbol{r}_{N}, t\right)$ is the $N$ particle probability density function, and $\boldsymbol{R}$ is a supervector defined by $\boldsymbol{R}=$ $\left(\boldsymbol{r}_{1}, \ldots, \boldsymbol{r}_{N}\right)$ with $\boldsymbol{r}_{i}$ the position vector of the particle $i$. In addition, $\hat{\mathcal{L}}$ is the Smoluchowski operator for the interacting Brownian particles without the HI, and it is given by

$$
\hat{\mathcal{L}}(\ldots)=\sum_{i=1}^{N} D_{i} \nabla_{i} \cdot\left\{\beta\left[\nabla_{i} \Phi(\boldsymbol{R})\right](\ldots)+\nabla_{i}(\ldots)\right\},
$$

where $\nabla_{i}$ is the gradient operator in terms of $\boldsymbol{r}_{i},(\ldots)$ represents a function on which $\hat{\mathcal{L}}$ operates, and $\beta$ is the inverse temperature $\beta=1 / k_{B} T$. The function $\Phi(\boldsymbol{R})$ is the total potential energy of interaction between the $N$ particles.

\section{B. Two-particle steady Smoluchowski equation}

Here, we derive a steady solution $P_{12}\left(\boldsymbol{r}_{1}, \boldsymbol{r}_{2}\right)$ of the twoparticle Smoluchowski equation under the condition that an external force $\boldsymbol{F}_{\text {ext }}$ is applied only to the particle 1. Let us denote the difference of the two position vectors as $\boldsymbol{r}:=\boldsymbol{r}_{1}-$ $\boldsymbol{r}_{2}$, and the potential energy of the two particles as $V_{12}(r)$ with $r=|\boldsymbol{r}|$. Setting $N=2$, and replacing $\nabla_{1} \Phi(\boldsymbol{R})$ and $\nabla_{2} \Phi(\boldsymbol{R})$ in Eq. (2) with $\nabla_{1} V_{12}(r)-\boldsymbol{F}_{\text {ext }}$ and $\nabla_{2} V_{12}(r)$, respectively, we obtain the steady two-particle Smoluchowski equation

$$
\nabla \cdot\left(\frac{\nabla P_{12}}{\beta}+P_{12} \nabla V_{12}-\frac{a_{2}}{2 a_{12}} P_{12} \boldsymbol{F}_{\text {ext }}\right)=0,
$$

where $\boldsymbol{\nabla}$ is the gradient operator in terms of $\boldsymbol{r}$, and $a_{12}$ is the mean radius $a_{12}:=\left(a_{1}+a_{2}\right) / 2$. In the derivation of Eq. (3), we use $\nabla=\nabla_{1}=-\nabla_{2}$, and $D_{i}=k_{B} T /\left(6 \pi \eta a_{i}\right)$.
If we assume that the external force $\boldsymbol{F}_{\text {ext }}$ is weak, then the solution of Eq. (3) to the first order in $\boldsymbol{F}_{\text {ext }}$ is given by [15]

$$
P_{12}(\boldsymbol{r})=P_{12}^{(0)}(r)\left[1+\beta a_{12} L_{12}(r) \hat{\boldsymbol{r}} \cdot \boldsymbol{F}_{\text {ext }}\right],
$$

where $P_{12}^{(0)}(r)$ is the equilibrium distribution $P_{12}^{(0)}(r) \propto$ $e^{-\beta V_{12}(r)}, L_{12}(r)$ is an unknown function to be specified, and $\hat{\boldsymbol{r}}$ is the unit vector in the direction of $\boldsymbol{r}$. Note that $a_{12}$ is introduced to make $L_{12}(r)$ nondimensional. Inserting Eq. (4) into Eq. (3) and neglecting the terms of $O\left(\boldsymbol{F}_{\text {ext }}^{2}\right)$, we obtain an equation for $L_{12}(r)$ as

$$
e^{-\beta V_{12}} \nabla \cdot \boldsymbol{q}_{12}(\boldsymbol{r})+\left(\nabla e^{-\beta V_{12}}\right) \cdot \boldsymbol{q}_{12}(\boldsymbol{r})=0,
$$

where the function $\boldsymbol{q}_{12}(\boldsymbol{r})$ is defined by

$$
\boldsymbol{q}_{12}(\boldsymbol{r}):=2 a_{12}^{2} \nabla\left[L_{12}(r) \hat{\boldsymbol{r}} \cdot \boldsymbol{F}_{\mathrm{ext}}\right]-a_{2} \boldsymbol{F}_{\mathrm{ext}} .
$$

For the hard-sphere interaction potential, we have $e^{-\beta V_{12}(r)}=1$ for $r>2 a_{12}$, and thus the second term in Eq. (5) vanishes. Therefore, we have

$$
\nabla^{2}\left[L_{12}(r) \cos \theta\right]=0 \quad\left(r>2 a_{12}\right),
$$

where we postulate that $\boldsymbol{F}_{\text {ext }}$ is parallel to the $z$ axis [i.e., $\left.\boldsymbol{F}_{\text {ext }}=\left(0,0, F_{\text {ext }}\right)\right]$, and $\theta$ is the angle between $\boldsymbol{r}$ and the $z$ axis. The Laplace equation (7) can be readily solved by expressing the Laplacian with spherical coordinates, and we obtain $L_{12}(r)=C / r^{2}$, where $C$ is a constant.

The constant $C$ can be determined with a boundary condition. In fact, by integrating Eq. (5) over an infinitesimal interval $\left(2 a_{12}-\epsilon, 2 a_{12}+\epsilon\right)$, the first term in Eq. (5) vanishes as $\epsilon \rightarrow 0$; integration of the second term can be carried out by using $\nabla e^{-\beta V_{12}(r)}=\hat{\boldsymbol{r}} \delta\left(r-2 a_{12}\right)$. Thus, Eq. (5) is rewritten as

$$
\left.2 a_{12}^{2} \frac{\partial L_{12}(r)}{\partial r}\right|_{r=2 a_{12}}-a_{2}=0,
$$

where $\hat{\boldsymbol{r}} \cdot \nabla=\partial / \partial r$ is used. Inserting $L_{12}(r)=C / r^{2}$ into Eq. (8), we obtain $C=-2 a_{12} a_{2}$. Thus, we have

$$
L_{12}(r)=-\frac{2 a_{2} a_{12}}{r^{2}},
$$

and obtain a steady solution [Eq. (4)] of the two-particle Smoluchowski equation (3).

\section{Long-time self-diffusion}

Using the steady solution obtained in the previous subsection, here we derive the long-time self-diffusion coefficient for the $N$-particle system. Suppose that the constant external force $\boldsymbol{F}_{\text {ext }}$ is exerted only on the particle $\boldsymbol{r}_{1}$. Then, the mean resultant force is balanced as $\gamma_{1}\left\langle\boldsymbol{v}_{1}\right\rangle=\boldsymbol{F}_{\text {ext }}-\left\langle\nabla_{1} \Phi\right\rangle-k_{B} T\left\langle\nabla_{1} \ln P\right\rangle$, where $v_{1}$ and $\gamma_{1}=1 / \beta D_{1}$ are the velocity and friction coefficient for the particle 1 , respectively. The second term is the force exerted by the other particles and the third term is the Brownian force. Thus, the mean drift velocity is given by

$$
\left\langle\boldsymbol{v}_{1}\right\rangle=\beta D_{1} \boldsymbol{F}_{\text {ext }}-\beta D_{1}\left\langle\nabla_{1} \Phi\right\rangle-D_{1}\left\langle\nabla_{1} \ln P\right\rangle .
$$

It should be noted that the ensemble average $\langle\ldots\rangle$ is taken in terms of the nonequilibrium steady state since the external force $\boldsymbol{F}_{\text {ext }}$ is applied.

Let us begin with the calculation of the second term on the right side of Eq. (10). We assume that the interaction potential is given by a sum of pair potentials 
$\Phi(\boldsymbol{R})=\sum_{i=1}^{N} \sum_{j=i+1}^{N} V_{i j}\left(r_{i j}\right)$, where $\boldsymbol{r}_{i j}=\boldsymbol{r}_{i}-\boldsymbol{r}_{j}$ and $r_{i j}=$ $\left|\boldsymbol{r}_{i j}\right|$ (the subscript $i j$ is necessary for the pair potential $V_{i j}$ because $V_{i j}$ depends on the particles radii $a_{i}$ and $a_{j}$ for the hard-sphere interaction). Then, we have

$$
\begin{aligned}
-\beta D_{1}\left\langle\nabla_{1} \Phi\right\rangle & \approx-\beta D_{1} \sum_{k=1}^{S} n_{k}\left\langle\nabla_{1} V_{1 N_{k}}\left(r_{1 N_{k}}\right)\right\rangle \\
& =-\beta D_{1} \sum_{k=1}^{S} n_{k} \int \cdots \int d \boldsymbol{R} P(\boldsymbol{R}) \nabla V_{1 N_{k}}\left(r_{1 N_{k}}\right) \\
& =-\beta D_{1} \sum_{k=1}^{S} n_{k} V \int d \boldsymbol{r} P_{1 N_{k}}(\boldsymbol{r}) \nabla V_{1 N_{k}}(r),
\end{aligned}
$$

where, in the first equality, we use an approximation $n_{1}-1 \approx$ $n_{1}$; in the second line, $P(\boldsymbol{R})$ is a steady solution of the $N$ particle Smoluchowski equation; and, in the third line, $V$ is the system volume and $\boldsymbol{r}:=\boldsymbol{r}_{1 N_{k}}$. Here, $P_{1 N_{k}}(\boldsymbol{r})$ in Eq. (11) is approximated by the steady solution for the two-particle Smoluchowski equation [Eq. (4)] with $P_{1 N_{k}}^{(0)}(r)=e^{-\beta V_{1 N_{k}}(r)} / V^{2}$ [15]. Then, by using $-\beta e^{-\beta V_{1 N_{k}}(r)} \nabla V_{1 N_{k}}(r)=\hat{\boldsymbol{r}} \delta\left(r-2 a_{1 N_{k}}\right)$ as well as $\int d \Omega \hat{\boldsymbol{r}}=0$ and $\int d \Omega \hat{\boldsymbol{r}} \hat{\boldsymbol{r}}=4 \pi \boldsymbol{I} / 3$ with $\boldsymbol{I}$ being the identity matrix and $\int d \Omega(\ldots)$ being the integration over the solid angle, we obtain

$$
-\beta D_{1}\left\langle\nabla_{1} \Phi\right\rangle \approx-\beta D_{1} \boldsymbol{F}_{\mathrm{ext}} K_{1}^{\mathrm{I}} \phi,
$$

where $K_{s}^{\mathrm{I}}$ is called a mobility coefficient [17], and is defined for $s=1, \ldots, S$ as

$$
K_{s}^{\mathrm{I}}:=2 \frac{\sum_{k=1}^{S} n_{k} \tilde{a}_{s k}^{2} \tilde{a}_{k}}{\sum_{k=1}^{S} n_{k} \tilde{a}_{k}^{3}} .
$$

Here, we define a mean radius $\tilde{a}_{s k}$ as $\tilde{a}_{s k}:=a_{N_{s} N_{k}}$.

Similarly, the third term on the right side of Eq. (10) is rewritten with the $N$-particle correlation function $g^{N}(\boldsymbol{R}):=$ $V^{N} P(\boldsymbol{R})$ as

$$
\begin{aligned}
-D_{1}\left\langle\nabla_{1} \ln P\right\rangle & =-D_{1}\left\langle\nabla_{1} \ln g^{N}\right\rangle \\
& \approx-D_{1} \sum_{k=1}^{S} n_{k}\left\langle\nabla_{1} \ln g_{1 N_{k}}\left(\boldsymbol{r}_{1 N_{k}}\right)\right\rangle,
\end{aligned}
$$

where we use the Kirkwood superposition approximation $g^{N}(\boldsymbol{R}) \approx \prod_{i=1}^{N} \prod_{j=i+1}^{N} g_{i j}\left(\boldsymbol{r}_{i j}\right)$ [33], where $g_{i j}(\boldsymbol{r})$ is the twoparticle correlation function for the particles $i$ and $j$. In the same way as Eq. (11), the ensemble average in Eq. (14) is rewritten as

$$
\left\langle\nabla_{1} \ln g_{1 N_{k}}\left(\boldsymbol{r}_{1 N_{k}}\right)\right\rangle=V \int d \boldsymbol{r} \nabla P_{1 N_{k}}(\boldsymbol{r}) .
$$

This integral can be calculated with Eqs. (4) and (9), and it is found to be vanishing. Thus, we have the third term in Eq. (10) as

$$
-D_{1}\left\langle\nabla_{1} \ln P\right\rangle \approx 0
$$

and the mobility coefficient for the Brownian force is vanishing, $K_{1}^{\mathrm{B}}=0$, for the case without the HI.

Therefore, Eq. (10) is now given by

$$
\left\langle\boldsymbol{v}_{1}\right\rangle=\beta D_{1}\left(1-K_{1}^{\mathrm{I}} \phi\right) \boldsymbol{F}_{\text {ext }} .
$$

From this equation, we obtain an effective friction constant $\gamma_{\text {eff }}$ as $\gamma_{\text {eff }}=\left[\beta D_{1}\left(1-K_{1}^{I} \phi\right)\right]^{-1}$. Furthermore, using the Einstein relation $D_{1}(\phi)=k_{\mathrm{B}} T / \gamma_{\mathrm{eff}}$, we then have the long-time self-diffusion coefficient for the particle $\boldsymbol{r}_{1}$ as

$$
D_{1}(\phi)=D_{1}(0)\left(1-K_{1}^{\mathrm{I}} \phi\right) .
$$

Now, it is obvious that the diffusion coefficient for the species $s$, which is denoted by $\tilde{D}_{s}(\phi)$, is given by

$$
\tilde{D}_{s}(\phi)=\tilde{D}_{s}(0)\left(1-K_{s}^{\mathrm{I}} \phi\right)
$$

for $s=1, \ldots, S$. It is also possible to express the diffusion coefficient $\tilde{D}_{s}(\phi)$ as a function of the volume fractions of the species $\phi_{k}(k=1, \ldots, S)$ as in Refs. [17,18]. Here, however, the expression as Eq. (19) is preferred because this paper focuses on dependence of the diffusivity on the total volume fraction $\phi$, and the composition of species is fixed. Setting $\tilde{a}_{s}=\tilde{a}_{s k}=a$ in Eq. (13), we obtain the results for identical particles as $\tilde{D}_{s}(\phi)=\tilde{D}_{s}(0)(1-2 \phi)$, and thus we recover the result presented in Refs. [15,21].

\section{Obstructed diffusion}

It would be interesting to compare the formula in Eqs. (13) and (19) to simulation results for obstructed diffusion, which is frequently employed to study crowding effect on intracellular diffusion [30,31]. Here, we suppose that there are only two species, a tagged particle and obstacles, i.e., $S=2$. Moreover, let us assume that there is only one tagged particle $n_{1}=1$, and many obstacles $n_{2} \gg 1$. Then, Eq. (13) for the tagged particle is rewritten as

$$
K_{1}^{I} \approx \frac{\left(\tilde{a}_{1}+\tilde{a}_{2}\right)^{2}}{2 \tilde{a}_{2}^{2}} .
$$

Equation (20) has an obvious physical meaning: the numerator is proportional to the scattering cross section between the tagged particle and an obstacle, and the denominator is proportional to the square of mean interobstacle distance with the volume fraction $\phi$ kept fixed. Therefore, the right side of Eq. (20) is proportional to the collision rate when the tagged particle travels the mean interobstacle distance. For small obstacles (i.e., for small $\tilde{a}_{2}$ ), the mean interobstacle distance becomes short, and therefore the collision rate becomes large. For large obstacles (i.e., for large $\tilde{a}_{2}$ ), both cross section and mean interobstacle distance become large, and therefore the collision rate tends to a minimum, and accordingly we have $K_{1}^{I} \rightarrow \frac{1}{2}$.

In Fig. 1, the reduction rate of the tagged particle $\tilde{D}_{1}(\phi) / \tilde{D}_{1}(0)$ is displayed for four different values of $\tilde{a}_{2} / \tilde{a}_{1}$. The numerical results (symbols) and the theoretical predictions (lines) given by Eqs. (19) and (20) are consistent even at relatively large volume fractions. It is found that, for a fixed volume fraction $\phi$, the reduction is greater for smaller obstacles. Note that these results are qualitatively similar to simulation results presented in Ref. [30], in which the obstructed diffusion is intensively studied with lattice models.

\section{E. Application to Ando-Skolnick model}

Here, let us apply the theoretical prediction obtained in the previous subsection to the Ando-Skolnick model. The Ando-Skolnick model consists of spheres with 15 different 


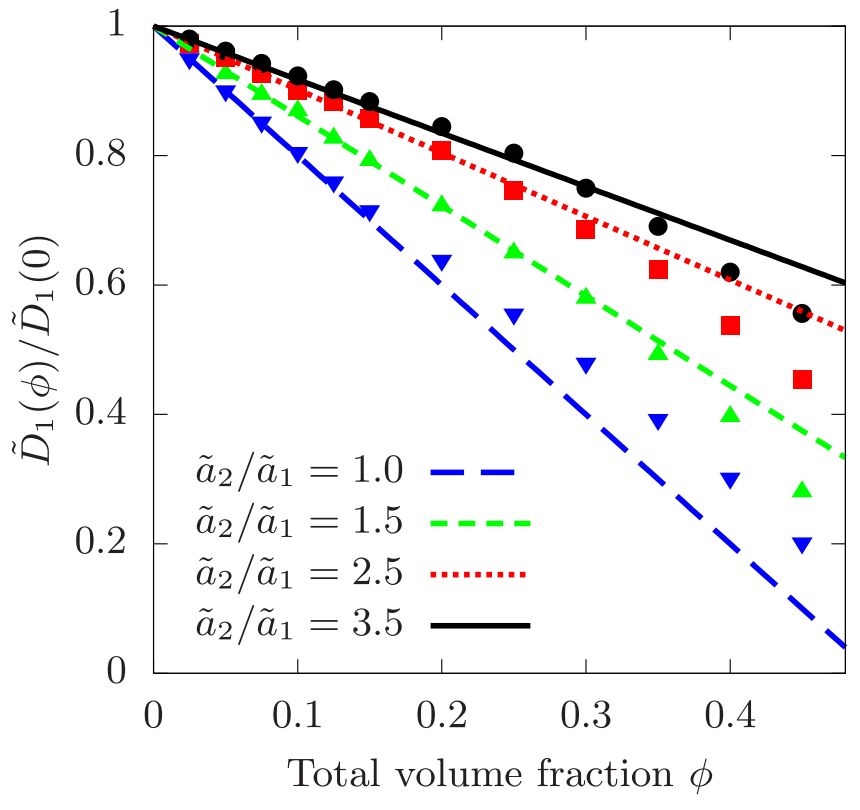

FIG. 1. Reduction rate $\tilde{D}_{1}(\phi) / \tilde{D}_{1}(0)$ of long-time diffusion coefficient vs total volume fraction $\phi$ for a tagged particle in obstructed diffusion (without the HI). It is assumed that there are only two species: the tagged particle $(s=1)$ and obstacles $(s=2)$. The ratio $\tilde{a}_{2} / \tilde{a}_{1}$ between the sizes of these two species is set as $\tilde{a}_{2} / \tilde{a}_{1}=1.0$ (triangle down), 1.5 (triangle up), 2.5 (square), and 3.5 (circle). The symbols are simulation results obtained with the numerical scheme in Appendix A; the lines are the theoretical predictions [Eq. (19)] with $K_{1}^{\mathrm{I}}$ given by Eq. (20)

sizes; these spherical particles are models of real molecules whose names are listed in Table I. For each species $s(s=$ $1, \ldots, 15)$, the number of particles $n_{s}$, the diffusion coefficient at infinite dilution $\tilde{D}_{s}$, and the radii $\tilde{a}_{s}$ are also given in Table I. The number of particles $n_{s}$ is determined so that the size distribution mimics that of real cytoplasm of E.coli $[10,11]$. The mobility coefficient $K_{s}^{\mathrm{I}}$ for the Ando-Skolnick model is calculated by inserting the values of $n_{s}$ and $\tilde{a}_{s}$ into Eq. (13), and the results thus obtained are presented in Table I.

In Fig. 2(a), theoretical predictions [Eq. (19)] of diffusion coefficients $\tilde{D}_{s}(\phi) / \tilde{D}_{s}(0)$ as functions of the volume fraction $\phi$ are displayed with lines. Similarly, in Fig. 3(a), $\tilde{D}_{s}(\phi) / \tilde{D}_{s}(0)$ is plotted as a function of the particle radius $\tilde{a}_{s}$. As can be seen from these figures, the reduction of diffusivity is more prominent for large particles than for small particles. By recalling the implications obtained in Fig. 1, the reason for these results can be understood as follows. If a tagged particle is small, surrounding particles (obstacles) are relatively large; these large obstacles result in a low collision rate of the tagged particle with the obstacles, and thereby the diffusivity reduces mildly as is the case for the GFP in Fig. 2(a). If the tagged particle is large, however, the surrounding particles (obstacles) are relatively small; these small obstacles lead to a high collision rate, and the diffusivity reduces rapidly as is the case for the ribosome in Fig. 2(a).

At low concentrations, the theoretical results are consistent with the numerical results displayed with symbols in Figs. 2(a) and 3(a). For large particles, however, huge deflections are observed at high concentrations; these de-
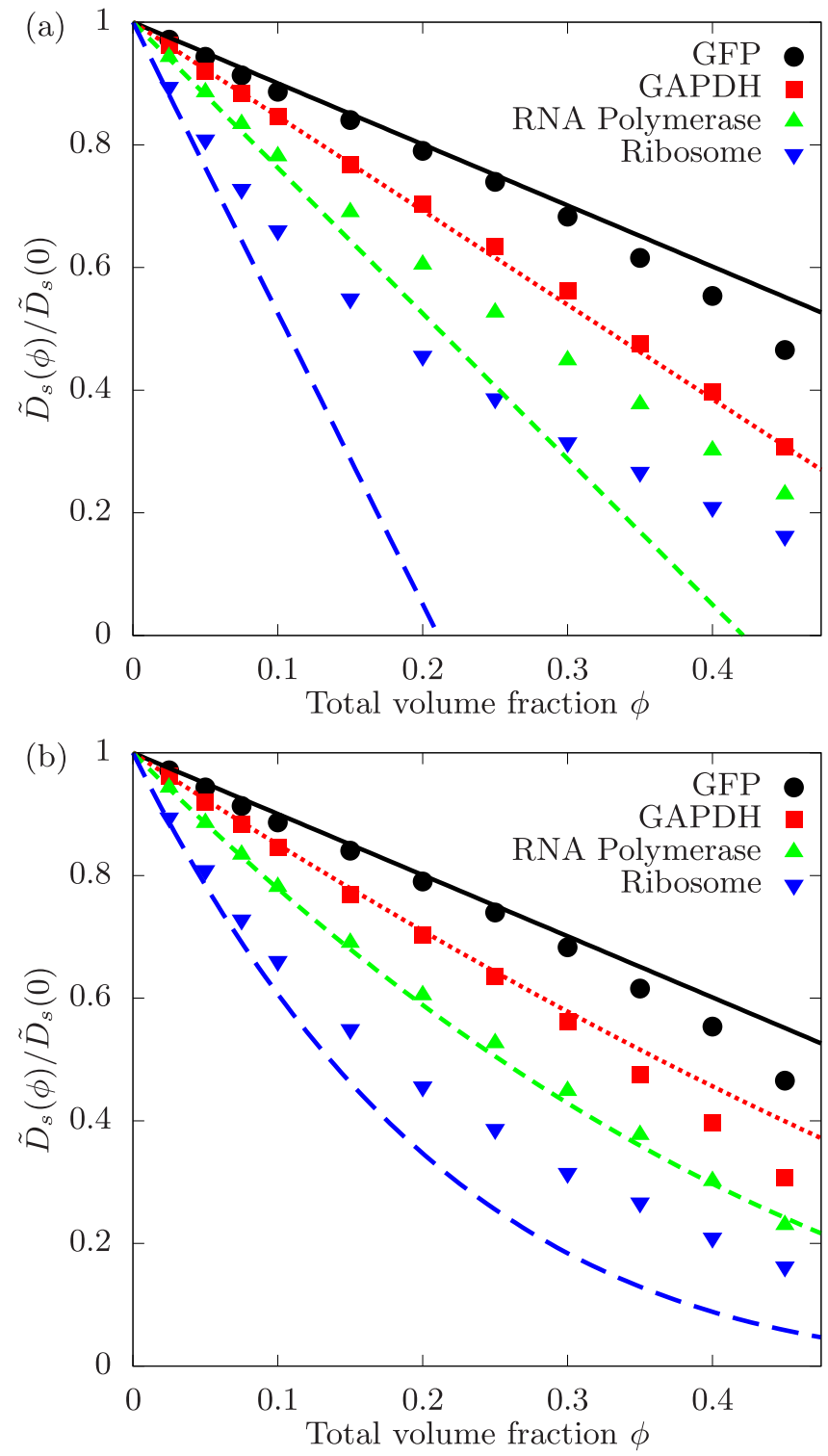

FIG. 2. Reduction rate $\tilde{D}_{s}(\phi) / \tilde{D}_{s}(0)$ of long-time diffusion coefficient vs total volume fraction $\phi$ for the Ando-Skolnick model without the HI. The results for four kinds of molecules are displayed: GFP (circle), GAPDH (square), RNA polymerase (triangle up), and ribosome (triangle down). The symbols are simulation results obtained with numerical scheme in Appendix A. [The data shown with the symbols in (a) and (b) are the same.] The lines are theoretical prediction obtained (a) with Eq. (19), and (b) with Eq. (23), where the values of $K_{s}^{\mathrm{I}}$ are given in Table I. The solid lines are the predictions for GFP, the dotted lines for GAPDH, the dashed lines for RNA polymerase, and the long-dashed lines for ribosome.

flections are due to the fact that the above theory is valid only for the first order in $\phi$, and higher-order contributions are neglected. The higher-order corrections for monodisperse suspensions without the HI are studied in Refs. [34,35]. Here, however, let us improve the above linear theory in a much simple and heuristic way, which is similar to the method used in Ref. [36].

When a volume $V \phi$ in the total free volume $V$ is filled by the particles, the diffusion coefficient is reduced with the rate 

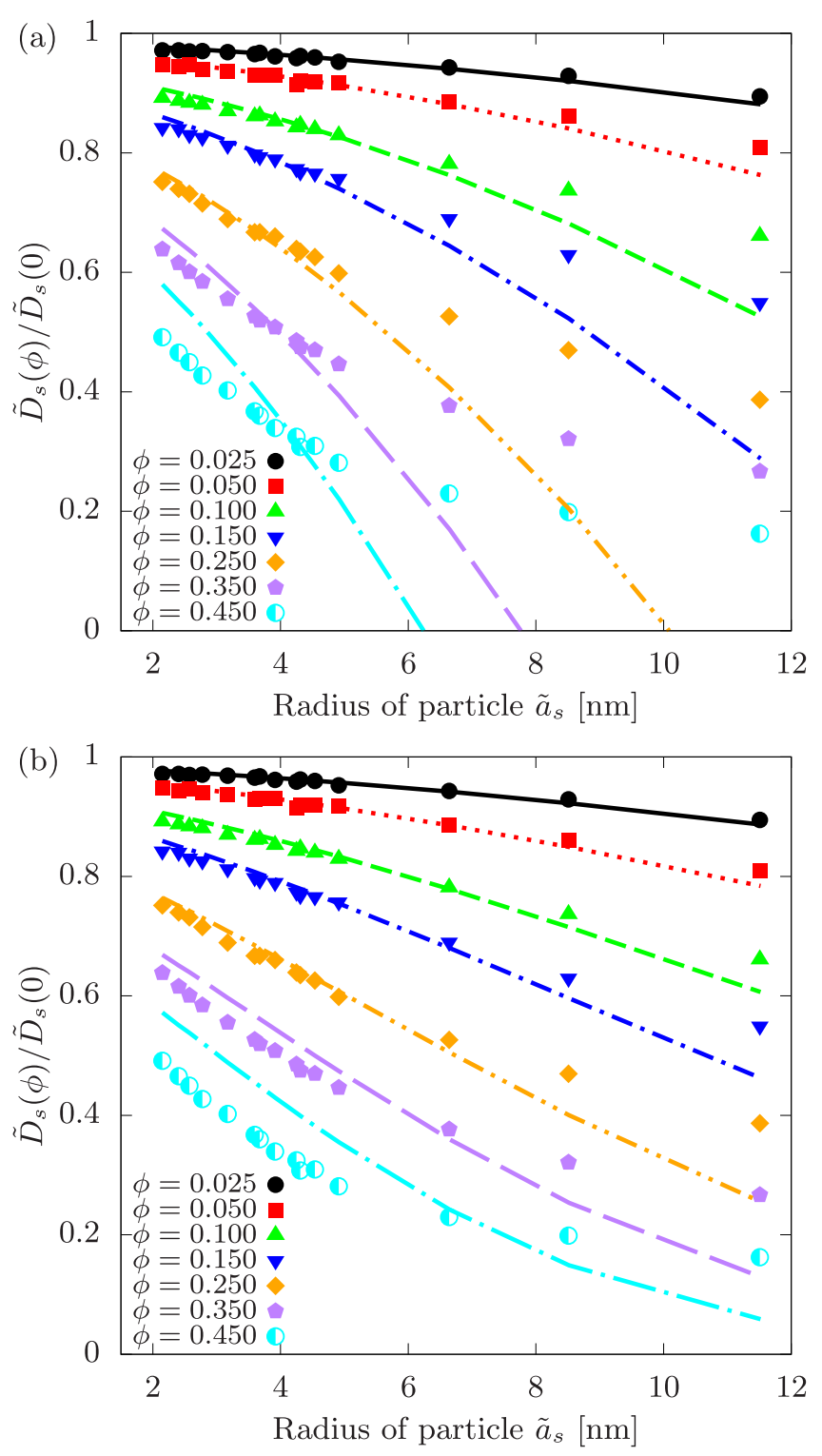

FIG. 3. Reduction rate $\tilde{D}_{s}(\phi) / \tilde{D}_{s}(0)$ of long-time diffusion coefficient vs radius of molecule $\tilde{a}_{s}$ for the Ando-Skolnick model without the HI. Numerical results for seven different values of the volume fraction $\phi$ are displayed: $\phi=0.025,0.05,0.1,0.15,0.25,0.35$, and 0.45 (from top to bottom). The lines are the corresponding theoretical predictions obtained (a) with Eq. (19) and (b) with Eq. (23).

$\tilde{D}_{s}(\phi) / \tilde{D}_{s}(0)=\tilde{D}_{s}(V \phi / V) / \tilde{D}_{s}(0)$. If the volume $V \phi$ is filled, then the remaining free volume is $V(1-\phi)$. Suppose that a volume $V d \phi$ in this free volume $V(1-\phi)$ is further filled. Here, the reduction rate $\tilde{D}_{s}(\phi+d \phi) / \tilde{D}_{s}(\phi)$ is assumed to be given by the same formula as the dilute limit:

$$
\frac{\tilde{D}_{s}(\phi+d \phi)}{\tilde{D}_{s}(\phi)} \approx \frac{\tilde{D}_{s}\left(\frac{V d \phi}{V(1-\phi)}\right)}{\tilde{D}_{s}(0)}=\frac{\tilde{D}_{s}\left(\frac{d \phi}{1-\phi}\right)}{\tilde{D}_{s}(0)} .
$$

Setting $d \phi \rightarrow 0$, we have an ordinary differential equation as

$$
\frac{d \tilde{D}_{s}(\phi)}{d \phi}=-K_{s}^{\mathrm{I}} \frac{\tilde{D}_{s}(\phi)}{1-\phi} .
$$

This equation is readily solved, and we obtain a heuristic theory as

$$
\tilde{D}_{s}(\phi)=\tilde{D}_{s}(0)(1-\phi)^{K_{s}^{1}}
$$

for $s=1, \ldots, S$. In contrast to the linear theory given by Eq. (19), for which the diffusion coefficient could be negative, in this modified theory [Eq. (23)], the diffusion coefficient is always positive, and thus it can be applied at least formally to systems at high concentrations. This modified theory is depicted in Figs. 2(b) and 3(b), and it is found that, for large particles, the modified theory is more consistent with the numerical results than the linear theory [Eq. (19)].

\section{WITH HYDRODYNAMIC INTERACTION}

In addition to the excluded volume effect, the $\mathrm{HI}$ is also taken into account in this section. In general, the motion of a Brownian particle creates a velocity field in the solvent, and this velocity field exerts forces on other Brownian particles; this type of interaction between particles is called the HI $[15,37]$. In many cases, the effect of the HI cannot be neglected to describe the dynamics of assembly of Brownian particles $[11,38,39]$. Here, we incorporate the HI through a far-field approximation of mobility functions, thereby obtaining a steady solution of the Smoluchowski equation. Once the far-field approximations of the mobility functions and the steady solution are obtained, the reduced diffusivity for polydisperse colloidal suspensions can be calculated by a procedure developed by Batchelor [16-18].

In the following subsection, far-field approximations of the mobility functions are given up to $O\left(1 / r^{7}\right)$ for an illustration. However, in obtaining the main results of this paper summarized in Table I, far-field approximations of $O\left(1 / r^{100}\right)$ are utilized for the mobility functions; this approximation is numerically obtained by a twin-multipole expansion for the Stokes equation and resulting recursion relations [14,22]. A steady solution of the Smoluchowski equation is then obtained numerically up to $O\left(1 / r^{99}\right)$.

By using these mobility functions and steady solution (more precisely, their series expansions), the reduced diffusivity is obtained with the Batchelor's method. This analysis is outlined in Appendix C, where a formula for the reduced diffusivity is given explicitly by using the mobility functions of $O\left(1 / r^{7}\right)$. In contrast, theoretical predictions for the reduced diffusivity of the Ando-Skolnick model (summarized in Table I) are numerically obtained by using the twin-multipole expansions up to $O\left(1 / r^{100}\right)$. The explicit expressions given in Appendix $\mathrm{C}$ with the accuracy of $O\left(1 / r^{7}\right)$ were used to check the correctness of this numerical scheme.

\section{A. Hydrodynamic interaction}

The Smoluchowski equation for a suspension with the HI is again given by Eq. (1), but the Smoluchowski operator $\hat{\mathcal{L}}$ should be replaced by [15]

$$
\hat{\mathcal{L}}(\ldots):=\sum_{i, j=1}^{N} \nabla_{i} \cdot \boldsymbol{D}_{i j} \cdot\left\{\beta\left[\nabla_{j} \Phi(\boldsymbol{R})\right](\ldots)+\nabla_{j}(\ldots)\right\} .
$$


The $(3 \times 3)$-dimensional matrices $\boldsymbol{D}_{i j}$ in Eq. (24) are the diffusion tensors (or $\beta \boldsymbol{D}_{i j}$ are usually referred to as mobility tensors), which represent the effect of the HI between particles $i$ and $j$. In fact, the inside of the curly brackets in Eq. (24) is a direct force $-\beta \boldsymbol{F}_{j}$ (i.e., it is the sum of potential and Brownian forces, but not the hydrodynamic force) exerted on the particle $j$, and then $\boldsymbol{D}_{i j} \cdot\left(-\beta \boldsymbol{F}_{j}\right)$ represents the hydrodynamic effect on the particle $i$. [Strictly speaking, $\boldsymbol{D}_{i i} \cdot\left(-\beta \boldsymbol{F}_{i}\right)$ includes not only the hydrodynamic effect, but also the direct effect $D_{i} \boldsymbol{I} \cdot\left(-\beta \boldsymbol{F}_{i}\right)=-\beta D_{i} \boldsymbol{F}_{i}$. See Eq. (25).]

For $i=j$, the diffusion tensors are given by

$$
\boldsymbol{D}_{i i}=D_{i} \boldsymbol{I}+D_{i} \sum_{\substack{l=1 \\ l \neq i}}^{N}\left[A_{i l}^{\mathrm{s}}\left(r_{i l}\right) \hat{\boldsymbol{r}}_{i l} \hat{\boldsymbol{r}}_{i l}+B_{i l}^{\mathrm{s}}\left(r_{i l}\right)\left(\boldsymbol{I}-\hat{\boldsymbol{r}}_{i l} \hat{\boldsymbol{r}}_{i l}\right)\right]
$$

where $\boldsymbol{I}$ is the identity tensor of rank three. The first term on the right side gives exactly the same term encountered in Eq. (2), and thus it represents a direct effect (i.e., not the HI). The second term, however, is the hydrodynamic effect due to the velocity field which is generated by the direct force on the particle $i$, is reflected by the other particles $l \neq i$, and returns back to $i$. Moreover, $A_{i j}^{\mathrm{s}}(r)$ and $B_{i j}^{\mathrm{s}}(r)$ are called mobility functions $[16,40]$, and their superscripts "s" stand for "the self-part." These mobility functions are given up to $O\left(1 / r^{7}\right)$ as $[14-16,40]$

$$
\begin{gathered}
A_{i j}^{\mathrm{s}}(r)=-\frac{15}{4} \frac{a_{i} a_{j}^{3}}{r^{4}}-2 \frac{a_{i} a_{j}^{5}}{r^{6}}+\frac{15}{2} \frac{a_{i}^{3} a_{j}^{3}}{r^{6}}+O\left(\frac{1}{r^{8}}\right), \\
B_{i j}^{\mathrm{s}}(r)=-\frac{17}{16} \frac{a_{i} a_{j}^{5}}{r^{6}}+O\left(\frac{1}{r^{8}}\right),
\end{gathered}
$$

respectively.

Similarly, for $i \neq j$, the diffusion tensors $\boldsymbol{D}_{i j}$ are given by

$$
\boldsymbol{D}_{i j}=D_{j}\left[A_{i j}^{\mathrm{c}}\left(r_{i j}\right) \hat{\boldsymbol{r}}_{i j} \hat{\boldsymbol{r}}_{i j}+B_{i j}^{\mathrm{c}}\left(r_{i j}\right)\left(\boldsymbol{I}-\hat{\boldsymbol{r}}_{i j} \hat{\boldsymbol{r}}_{i j}\right)\right] .
$$

The diffusion tensor $\boldsymbol{D}_{i j}(i \neq j)$ represents a hydrodynamic effect on the particle $i$ due to the velocity field generated by the direct force on the particle $j$. The mobility functions $A_{i j}^{\mathrm{c}}(r)$ and $B_{i j}^{\mathrm{c}}(r)$, where the superscripts "c" stand for "the cross part," are given up to $O\left(1 / r^{7}\right)$ as [14-16,40]

$$
\begin{gathered}
A_{i j}^{\mathrm{c}}(r)=\frac{3}{2} \frac{a_{j}}{r}-\frac{a_{j}}{2} \frac{a_{i}^{2}+a_{j}^{2}}{r^{3}}+\frac{75}{4} \frac{a_{i}^{3} a_{j}^{4}}{r^{7}}+O\left(\frac{1}{r^{8}}\right), \\
B_{i j}^{\mathrm{c}}(r)=\frac{3}{4} \frac{a_{j}}{r}+\frac{a_{j}}{4} \frac{a_{i}^{2}+a_{j}^{2}}{r^{3}}+O\left(\frac{1}{r^{8}}\right) .
\end{gathered}
$$

We also use notations such as $\tilde{A}_{s k}^{\mathrm{s}}(r)$ and $\tilde{A}_{s k}^{\mathrm{c}}(r)$ to indicate that these are the mobility functions for the species $s$ and $k$. For example, $\tilde{A}_{s k}^{\mathrm{s}}(r)$ is defined by

$$
\tilde{A}_{s k}^{\mathrm{s}}(r)=-\frac{15}{4} \frac{\tilde{a}_{s} \tilde{a}_{k}^{3}}{r^{4}}-2 \frac{\tilde{a}_{s} \tilde{a}_{k}^{5}}{r^{6}}+\frac{15}{2} \frac{\tilde{a}_{s}^{3} \tilde{a}_{k}^{3}}{r^{6}}+O\left(\frac{1}{r^{8}}\right) .
$$

The other mobility functions for species are defined similarly.

The mobility tensor up to the third order $O\left(1 / r^{3}\right)$ is referred to as the Rotne-Prager-Yamakawa tensor $[41,42]$, which is frequently used in numerical simulations of colloidal suspensions $[43,44]$. The mobility tensor defined with Eqs. (25)-(30) describes only far-field two-body HI, but farfield many-body $\mathrm{HI}$ is also important to study concentrated systems. In fact, in Ref. [11], so-called Stokesian dynamics $[45,46]$ is employed as a simulation method in order to take into account the many-body HI. Moreover, in the Stokesian dynamics, a near-field two-body interaction called a lubrication effect is also taken into account.

Another, more simple, method to incorporate the manybody HI is employed in Refs. [13,47-49]. In these studies, the Brownian dynamics simulation is carried out with a theoretical prediction for the short-time diffusion coefficient given in Refs. [50,51], where the HI is approximately taken into account. Then, the Brownian dynamics simulation is carried out as if it is a hard-sphere colloidal suspension without the $\mathrm{HI}$; the $\mathrm{HI}$ is incorporated only through the short-time diffusivity. Also, it should be noted that the theoretical prediction for the short-time diffusivity in Refs. [50,51] is valid only for the monodisperse suspension and, therefore, in this numerical method, polydispersity is taken into account only through direct interaction (such as the hard-sphere interaction).

In Eqs. (25) and (28), only far-field two-body interactions are taken into account. In other words, HIs between three or more particles are neglected in this paper because these contributions are of the order of $O\left(\phi^{2}\right)$ [15]. We also assume the no-slip boundary condition [40].

\section{B. Reduction of diffusion coefficient}

Here, an outline of the calculation of the long-time selfdiffusion coefficient for polydisperse suspensions with the $\mathrm{HI}$ is presented. Details of this calculation are presented in Appendix C. The final result is that the long-time diffusion coefficient $\tilde{D}_{s}(\phi)$ for the species $s$ is given by

$$
\tilde{D}_{s}(\phi) \approx \tilde{D}_{s}(0)\left(1-K_{s}^{\mathrm{h}} \phi\right)
$$

for $s=1, \ldots, S$. Here, $K_{s}^{\mathrm{h}}$ is a total mobility coefficient for the species $s$ and it is defined by

$$
K_{s}^{\mathrm{h}}:=K_{s}^{\mathrm{hS}}+K_{s}^{\mathrm{hI}}+K_{s}^{\mathrm{hB}},
$$

where $K_{s}^{\mathrm{hS}}, K_{s}^{\mathrm{hI}}$, and $K_{s}^{\mathrm{hB}}$ are mobility coefficients concerning the short-time diffusivity, the hard-sphere interaction, and the Brownian force, respectively. In these definitions, the superscript " $h$ " stands for the HI. These mobility coefficients $K_{s}^{\mathrm{hS}}, K_{s}^{\mathrm{hI}}$, and $K_{s}^{\mathrm{hB}}$ are given explicitly up to $O\left(1 / r^{7}\right)$ in Appendix C [Eqs. (C30), (C42), (C48), and (C50)]. Moreover, the modified theory can also be obtained in the same way as in the case without the HI [see the derivation of Eq. (23)] as

$$
\tilde{D}_{s}(\phi)=\tilde{D}_{s}(0)(1-\phi)^{K_{s}^{\mathrm{h}}} \text {. }
$$

To calculate $K_{s}^{\mathrm{h}}(s=1, \ldots, S)$, first the far-field approximations of the mobility functions $A_{i j}^{\mathrm{s}}(r), A_{i j}^{\mathrm{c}}(r), B_{i j}^{\mathrm{s}}(r)$, and $B_{i j}^{\mathrm{c}}(r)$ are obtained by the twin-multipole expansion of the Stokes equation and resulting recursion relations [14,22]. [In Eqs. (26), (29), (27), and (30), expansions up to $O\left(1 / r^{7}\right)$ are given for reference. But, expansions of $O\left(1 / r^{100}\right)$ are used to obtain the data shown in Table I.]

Second, a series expansion for the steady solution of the Smoluchowski equation is derived. More precisely, the expansion coefficients $\alpha_{n}(n=1, \ldots, M)$ of $L_{12}(r)$ in Eq. (C15) are obtained by numerically solving a system of linear equations with $M-1$ variables $\alpha_{n}(n=1, \ldots, M-1) . M-2$ of these linear equations can be obtained by inserting Eq. (C15) into Eq. (C11) and making $M-2$ equations for the orders 
$1 / r^{n}(n=1,3, \ldots, M-1)$. The one remaining linear equation is obtained from a boundary condition given in Eq. (C22). [In obtaining the data in Table I, $M$ is set as $M=99$.]

Third, by using these series expansions of the mobility functions and the steady solution, the values of $K_{s}^{\mathrm{hS}}, K_{s}^{\mathrm{hI}}$, and $K_{s}^{\mathrm{hB}}$ are obtained. In fact, $K_{s}^{\mathrm{hS}}, K_{s}^{\mathrm{hI}}$, and $K_{s}^{\mathrm{hB}}$ are calculated by inserting the series expansions into the left side of Eq. (C28), the right sides of Eqs. (C42) and (C48), respectively. All of these calculations are performed numerically. Accordingly, the sum of these coefficients gives the total mobility coefficient $K_{s}^{\mathrm{h}}$, and thus we obtain the diffusion coefficient $\tilde{D}_{s}(\phi)$ of species $s$ at the concentration $\phi$ [Eq. (32)].

\section{Obstructed diffusion}

As an example, the two-species system explained in Fig. 1 is analyzed again, but here the HI is taken into account. Again, let us assume that there are only two species: the tagged particle $(s=1)$ and the obstacles $(s=2)$. By using the procedure explained in the previous subsection with $n_{1}=1$ and $n_{2} \gg n_{1}$, the values of the mobility coefficients $K_{s}^{\mathrm{hS}}, K_{s}^{\mathrm{hI}}$, $K_{s}^{\mathrm{hB}}$, and $K_{s}^{\mathrm{h}}$ are calculated for four different values of the ratio of radii: $\tilde{a}_{2} / \tilde{a}_{1}=1.0,1.5,2.5$, and 3.5 . Then, the diffusion coefficient for the tagged particle is obtained by Eqs. (32) and (33).

For the case with the HI, the most significant contribution to the reduction rate is from the leading term of the mobility coefficient $K_{1}^{\mathrm{hS}}$ [Eq. (C30)] as

$$
K_{1}^{\mathrm{hS}} \approx \frac{15}{4} \frac{1}{1+\tilde{a}_{2} / \tilde{a}_{1}} .
$$

This term arises from the leading term of the mobility function $A_{i j}^{\mathrm{s}}(r)$ [Eq. (26)]. Thus, the diffusivity reduction is mainly attributed to the first reflection of the velocity field at the obstacles. Moreover, this leading term of $K_{1}^{\mathrm{hS}}$ is in a finite range $(0,15 / 4)$, in contrast to $K_{1}^{\mathrm{I}}$ for the case without the HI [Eq. (20)], which is unbounded. Moreover, the mobility coefficient $K_{1}^{\mathrm{hB}}$ depends on the ratio $\tilde{a}_{2} / \tilde{a}_{1}$ almost oppositely. Namely, $K_{1}^{\mathrm{hS}}$ decreases with $\tilde{a}_{2} / \tilde{a}_{1}$; in contrast, $K_{1}^{\mathrm{hB}}$ increases with $\tilde{a}_{2} / \tilde{a}_{1}$, if the ratio is not too large. The contribution from the direct interaction $K_{1}^{\mathrm{hI}}$ is almost vanishing.

Therefore, it might follow that the reduction of diffusivity (characterized by $K_{1}^{\mathrm{h}}=K_{1}^{\mathrm{hS}}+K_{1}^{\mathrm{hI}}+K_{1}^{\mathrm{hB}}$ ) for the system with the $\mathrm{HI}$ is less dependent on the obstacle size than that for the system without the HI. In fact, this is the case as is clear from comparison of Figs. 1 and 4. This weak dependency on the obstacle size seems qualitatively consistent with a numerical simulation presented in a previous study (Fig. 8 in Ref. [48]), where the $\mathrm{HI}$ is incorporated by using a theoretical formula for the short-time diffusion coefficient given in Refs. [50,51].

However, there seems to be a qualitative discrepancy: the diffusion coefficient obtained by the present theory overestimates the numerical result presented in Ref. [48]. The range of volume fraction in Fig. 8 of Ref. [48] seems to be limited to relatively low concentration. [In that figure, $100 \mathrm{~g} / \mathrm{L}$ might correspond to $\phi=0.085$.] In this low concentration range, it is probable that our result would give a better estimate.

\section{Application to Ando-Skolnick model}

By using the procedure given in Sec. III B with the parameter values $n_{s}, \tilde{D}_{s}$, and $\tilde{a}_{s}$ listed in Table I, we obtain the

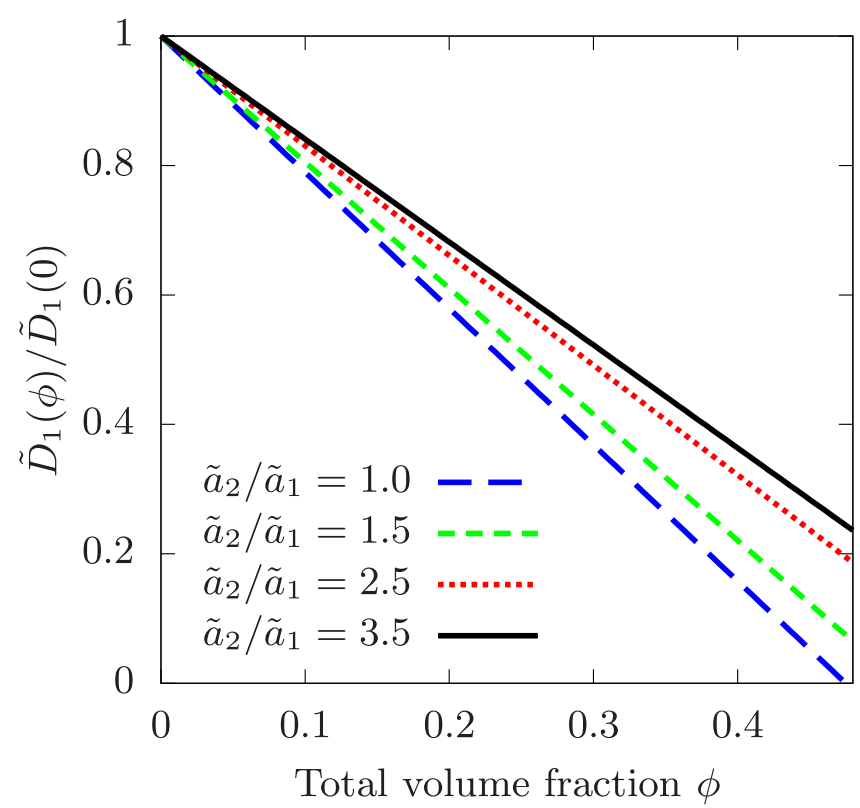

FIG. 4. Reduction rate $\tilde{D}_{1}(\phi) / \tilde{D}_{1}(0)$ of long-time diffusion coefficient vs total volume fraction $\phi$ for a tagged particle in obstructed diffusion (with the HI). It is assumed that there are only two species: the tagged particle $(s=1)$ and obstacles $(s=2)$. The ratio $\tilde{a}_{2} / \tilde{a}_{1}$ between the sizes of these two species is set as $\tilde{a}_{2} / \tilde{a}_{1}=1.0,1.5$, 2.5, and 3.5. The lines are the theoretical predictions [Eq. (32)] with $K_{1}^{\mathrm{h}}$ given by Eq. (33). The values of $K_{1}^{\mathrm{h}}$ are calculated by the procedure explained in Sec. III B with expansions of the mobility functions of $O\left(1 / r^{100}\right)$; for $\tilde{a}_{2} / \tilde{a}_{1}=1.0,1.5,2.5$, and 3.5 , we obtain $K_{1}^{\mathrm{h}}=2.11,1.95,1.70$, and 1.59 , respectively.

values of the mobility coefficients $K_{s}^{\mathrm{hS}}, K_{s}^{\mathrm{hI}}, K_{s}^{\mathrm{hB}}$, and $K_{s}^{\mathrm{h}}$ for the Ando-Skolnick model. These values, summarized also in Table I, are accurate up to $O\left(1 / r^{99}\right)$.

The mobility coefficient $K_{s}^{\mathrm{hS}}$ concerning the short-term diffusion is much greater than the other two coefficient $K_{s}^{\mathrm{hI}}$ and $K_{s}^{\mathrm{hB}}$. This term $K_{s}^{\mathrm{hS}}$ is due to purely hydrodynamic effect, and therefore absent for the case without the HI. In addition, this effect $\left(K_{s}^{\mathrm{hS}}\right)$ is greater for large particles than for small ones. This can be roughly understood with Eq. (35) as follows. For a large particle (with a radius $\tilde{a}_{1}$ ), surrounding particles are relatively small (with a mean radius $\tilde{a}_{2}<\tilde{a}_{1}$ ), and accordingly $\tilde{a}_{2} / \tilde{a}_{1}$ is small thereby resulting in a large value of $K_{1}^{\mathrm{hS}}$.

In contrast, the effect of the direct interaction $K_{s}^{\mathrm{hI}}$ is quite small. It is consistent with the results of near-field analysis which shows that this term is actually vanishing [22]. The effect of the Brownian force $K_{s}^{\mathrm{hB}}$ is also small, but its contribution to the reduction of the diffusivity is measurable. In addition, $K_{s}^{\mathrm{hB}}$ is greater for small particles than large ones; this correlation with the size is opposite to that of $K_{s}^{\mathrm{hS}}$. Note also that the effect of the Brownian force is absent for the case without the HI.

In Fig. 5(a), the reduction rate of diffusion coefficient $\tilde{D}_{s}(\phi) / \tilde{D}_{s}(0)$ is shown as a function of the volume fraction $\phi$. Comparing Fig. 5(a) with Fig. 2(a), it is found that variations of the reduction rates over particles with different sizes (with $\phi$ being fixed) is weaker for the system with the HI than for the system without the HI. In some sense, the HI plays a role of averaging the diffusion dynamics of particles with different 

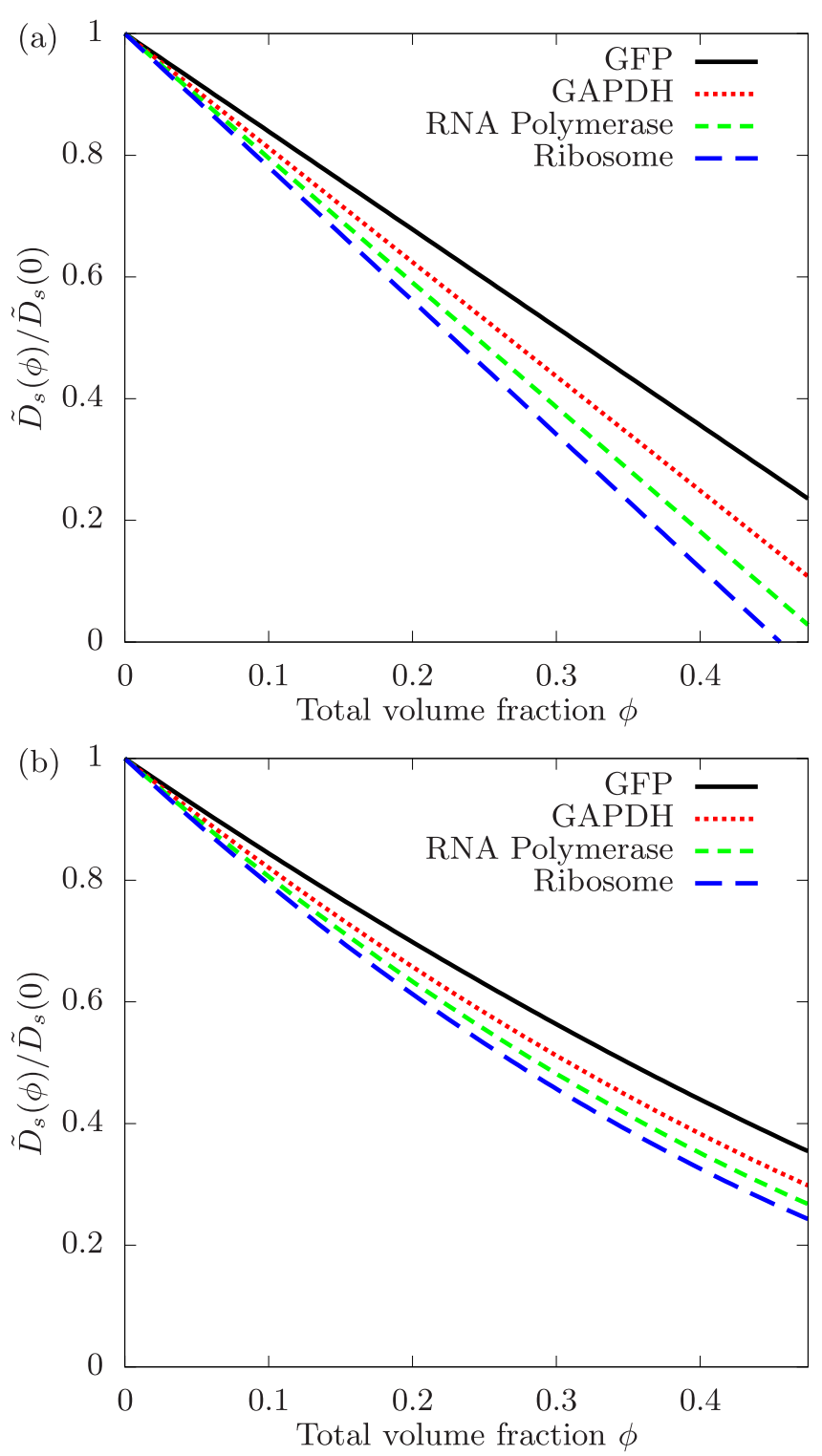

FIG. 5. Reduction rate $\tilde{D}_{s}(\phi) / \tilde{D}_{s}(0)$ of long-time diffusion coefficient vs total volume fraction $\phi$ for the system with the HI. The lines are theoretical prediction obtained (a) with Eq. (32) and (b) with Eq. (34). The solid lines are the predictions for GFP, the dotted lines for GAPDH, the dashed lines for RNA polymerase, and the long-dashed lines for ribosome.

sizes. This is more clearly seen from Fig. 6(a), in which the reduction rate is plotted as a function of the radius $\tilde{a}_{s}$; in fact, the curve at each concentration $\phi$ is flatter than that in Fig. 3(a).

Moreover, the mobility coefficient for most of the species shows large increases by incorporating the HI. This means that the diffusion coefficients for these species decrease by incorporating the HI. For example, the diffusion coefficient of the GFP is estimated as $D_{\mathrm{GFP}}(\phi) \simeq D_{\mathrm{GFP}}(0)(1-1.00 \phi)$ for the system without the HI, and by $D_{\mathrm{GFP}}(\phi) \simeq D_{\mathrm{GFP}}(0)(1-$ $1.61 \phi)$ for the system with the HI. This tendency agrees qualitatively with the results presented in Ref. [11].

Although there are approximated theories for the higherorder terms in $\phi$ [50-52], these theories are formulated for
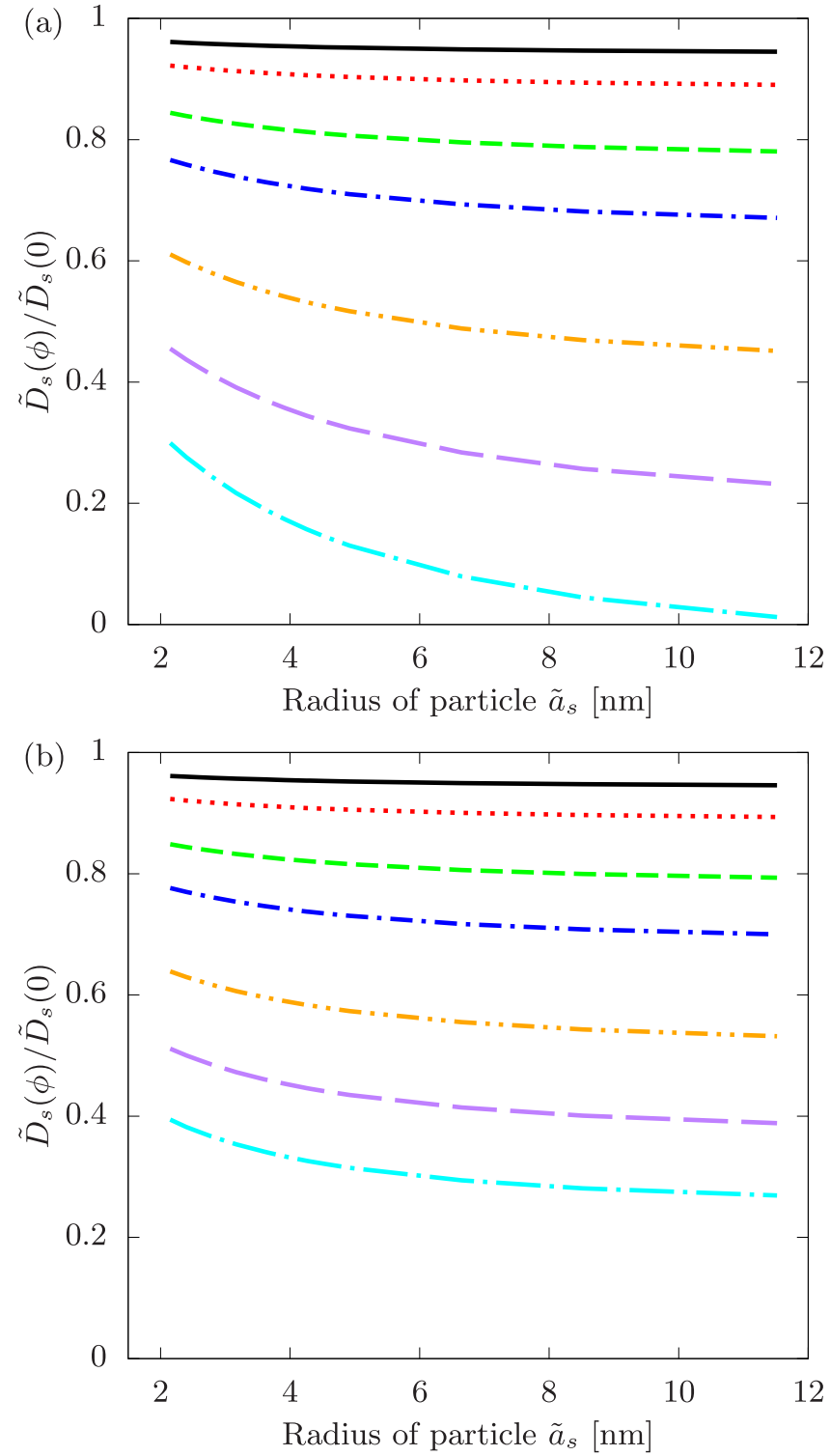

FIG. 6. Reduction rate $\tilde{D}_{s}(\phi) / \tilde{D}_{s}(0)$ of long-time diffusion coefficient vs radius of molecule $\tilde{a}_{s}$ for the Ando-Skolnick model with the HI. The lines are the theoretical predictions obtained (a) with Eq. (32) and (b) with Eq. (34). The results for seven different values of the volume fraction $\phi$ are displayed: $\phi=$ $0.025,0.05,0.1,0.15,0.25,0.35$, and 0.45 (from top to bottom).

monodisperse systems. Therefore, we again employ the modified theory given by Eq. (34) to study the Ando-Skolnick model, which is a polydisperse suspension. This modified theory is displayed in Figs. 5(b) and 6(b). As shown in these figures, differences between the modified theory [Eq. (34)] and the original theory [Eq. (32)] are smaller for the case with the HI than those for the case without the HI.

An interesting problem concerning diffusion in cytoplasm is how its concentration might be optimized; one of the possibilities is that it might be optimized to make chemical reactions as fast as possible. For example, in Refs. [53,54], a reaction rate of a single molecule is estimated by means of a theory of identical Brownian particles (i.e., the monodisperse suspension) in Ref. [55]. It was found that the optimal volume 
fraction for which the reaction rate attains its maximum is about $\phi^{*} \approx 0.18$; this value is much lower than the concentration in real cytoplasm. (For the Ando-Skolnick model, a concentration $300 \mathrm{mg} / \mathrm{mL}$ of macromolecules, which is a typical value for E.coli, corresponds to the volume fraction of $\phi \approx 0.51[11]$.)

However, the use of the theory of the identical Brownian particles seems difficult to be justified, and thus it is tempting to apply the present theory to the optimization problem in cytoplasm. Assuming that the reaction is diffusion limited, it can be shown that the reaction rate $R(\phi)$ is proportional to $D(\phi) \phi[56]$. If $D(\phi)=D(0)(1-K \phi)$ or $D(\phi)=D(0)(1-$ $\phi)^{K}$, where $K$ is the reduction coefficient, then the reaction rate $R(\phi)$ is maximized at $\phi^{*}=1 / 2 K$ or $\phi^{*}=1 /(K+1)$, respectively. For example, the maximum reaction rate for the GFP is attained at $\phi^{*} \approx 0.31$ or $\phi^{*} \approx 0.38$, respectively; these values are much closer to the realistic value $\phi=0.51$ than that given by the monodisperse theory (i.e., $\phi^{*} \approx 0.18$ ).

\section{DISCUSSION}

Reduction of diffusion coefficients of macromolecules in cytoplasm has been observed both in experiments [7,8] and numerical simulations of coarse-grained models of cytoplasm $[10,11,13]$. In particular, it was numerically found that the diffusion coefficient decreases significantly by incorporating the HI [11]. To fully understand what causes such a decrease in diffusivity, however, it is important to address the problem theoretically.

By applying a colloid theory [15-18] to the Ando-Skolnick model with and without the HI, this work presented an analytical prediction for the reduction of diffusivity up to the first order in concentration $\phi$ [Figs. 2(a) and 5(a)]. Moreover, to obtain predictions that are valid for more concentrated systems, heuristic modifications of these theories were developed [Figs. 2(b) and 5(b)]. In particular, it was found that the diffusion coefficients for small particles decrease prominently by incorporating the HI. For example, the diffusion coefficient of the GFP is estimated as $D_{\mathrm{GFP}}(\phi) \simeq D_{\mathrm{GFP}}(0)(1-1.00 \phi)$ for the system without the HI, and by $D_{\mathrm{GFP}}(\phi) \simeq D_{\mathrm{GFP}}(0)(1-$ $1.61 \phi)$ for the system with the HI. This is contrasting to the monodisperse systems for which the decrease of diffusivity is very small (see the Introduction).

In contrast, the diffusion coefficients for the three large particles such as the ribosomes increase by incorporating the HI, which is inconsistent with the results of Ref. [11]. Moreover, although the theoretical predictions for the diffusivity shown in Fig. 6(b) exhibit a trend qualitatively similar to simulation data reported in Ref. [11], the former is slightly larger than the latter. In addition, the reduction rate $D_{\mathrm{GFP}}(\phi) / D_{\mathrm{GFP}}(0)$ for the GFP at $\phi=0.51^{1}$ is about 0.32 in the theoretical prediction presented in Fig. 5(b); this prediction overestimates the experimental ob-

\footnotetext{
${ }^{1}$ At this value of the volume fraction, $\phi=0.51$, the concentration of the molecules is about $300 \mathrm{mg} / \mathrm{ml}$, which is close to the intracellular condition. The concentration of the Ando-Skolnick model can be calculated from the molecular weight of each molecule, which is given in Ref. [11].
}

servation of the reduction rate which is about 0.07-0.09 $[7,8]$. These differences should be mainly ascribed to the fact that applicability of the present theory is limited to the first order in concentration $\phi$. In numerical simulations reported in Ref. [11], many-body far-field interaction is taken into account with the Durlofsky-Brady-Bossis method [45], whereas, in this paper, only the two-body far-field interaction is taken into consideration. Moreover, in Ref. [11], near-field contributions of the $\mathrm{HI}$ are taken into account by a lubrication theory, whereas it is not considered here. These differences in the ways of incorporating the HI should be another source of the deflections observed.

Future work is therefore needed to extend the present theory to take into account contributions of higher orders in the concentration $\phi[34,35,50-52,57]$. Indeed, in this work, the two-particle correlation function $g_{i j}(\boldsymbol{r})$ is treated only to the leading order, but it is evident that the higher-order contributions must be included for analyzing highly concentrated systems.

Moreover, in many single-particle-tracking experiments, it has been reported that the diffusion coefficient shows large scatter [1-3,5,58]. Intensive theoretical efforts have also been devoted to explain such fluctuation of diffusivity, and simple stochastic models such as continuous-time random walk [28], fractional Brownian motion [59], random walk in fractal lattices [60,61], and random diffusivity models [62-64] were widely investigated through various time-series analysis methods $[28,39,65,66]$. In addition to the studies of these simple models, however, it would be important also to elucidate whether more realistic models, such as the Ando-Skolnick model, can describe such large scatter.

As stated in the Introduction, the transient subdiffusion is frequently observed in experiments and simulations, though we focus only on the long-time normal diffusion in this paper. The existence of such transient dynamics in the Ando-Skolnick model should be explored in future studies. Moreover, chemical reaction is also discussed at the end of Sec. III D through the mean first passage time. It might be necessary to take reaction kinetics into account directly in the coarse-grained model, if copy numbers of reactants are very small [12]. Finally, it is found that the diffusivity in the bacterial cytoplasm depends on the cellular metabolism [5]. Therefore, an important question for future studies is elucidation of effects of activeness such as active conformational changes of colloidal particles.

\section{ACKNOWLEDGMENT}

This work was supported by Grant-in-Aid (KAKENHI) for Scientific Research C (Grant No. JP18K03417).

\section{APPENDIX A: NUMERICAL SIMULATION OF HARD-SPHERE SUSPENSION WITHOUT HYDRODYNAMIC INTERACTION}

In this Appendix, we briefly review the numerical scheme proposed in Ref. [24] for Brownian hard spheres without the HI. For such systems, the equation of motion is given by the 
Langevin equation

$$
\frac{d \boldsymbol{r}_{i}}{d t}=\sqrt{2 D_{i}} \xi_{i}(t) \quad(i=1, \ldots, N),
$$

where $\boldsymbol{r}_{i}(t)$ is the position of the particle $i$, and $\boldsymbol{\xi}_{i}(t)$ is Gaussian white-noise vector which satisfies

$$
\begin{gathered}
\left\langle\boldsymbol{\xi}_{i}(t)\right\rangle=\mathbf{0}, \\
\left\langle\boldsymbol{\xi}_{i}(t) \boldsymbol{\xi}_{j}\left(t^{\prime}\right)\right\rangle=\boldsymbol{I} \delta_{i j} \delta\left(t-t^{\prime}\right),
\end{gathered}
$$

where $\boldsymbol{I}$ is the identity matrix of order three. The Euler method is used as an integration scheme of Eq. (A1) [67] with periodic boundary conditions, and the Mersenne twister method is employed as a pseudorandom number generator [68].

To take into account the hard-sphere interaction potential $\Phi\left(\boldsymbol{r}_{1}, \ldots, \boldsymbol{r}_{N}\right)$ [Eq. (2)], a simulation scheme of event-driven Brownian dynamics for polydisperse hard-sphere suspensions is employed [24]. In this scheme, Eq. (A1) is discretized as

$$
\boldsymbol{r}_{i}(t+\delta t)-\boldsymbol{r}_{i}(t)=\sqrt{2 D_{i} \delta t} \boldsymbol{\xi}_{i}(t),
$$

where $\delta t$ is the time to the next collision. Accordingly, it can be interpreted that each particle undergoes a linear uniform motion with a pseudovelocity $\boldsymbol{v}_{i}(t)=\sqrt{2 D_{i} / \delta t} \boldsymbol{\xi}_{i}(t)$ until $t+$ $\delta t$ at which two of the $N$ particles collide. Therefore, during this time interval $\delta t$, the particles follow classical Newtonian dynamics without any external force. It follows that the time to the next collision $\delta t$ can be determined in the same way as the classical hard-sphere system.

In addition, at every time step $\delta \tau$, the thermal noise $\boldsymbol{\xi}_{i}(t)$ and the pseudovelocity are updated. The value of $\delta \tau$ is determined so that the mean number of collisions for each particle during $\delta \tau$ is less than 1 . Thus, the time step $\delta \tau$ is shorter for systems with higher concentration. For example, for $\phi=$ 0.025 (the most dilute system in our simulation), we set $\delta \tau \approx 20 \mathrm{nsec}$, whereas, for $\phi=0.45$ (the most concentrated system), we set $\delta \tau \approx 0.1 \mathrm{nsec}$.

The collision dynamics is given as follows. Suppose that the particles $i$ and $j$ collide at time $t_{c}$, then the collision dynamics during a short-time interval which includes $t_{c}$ can be described by the two-particle diffusion equation

$$
\frac{\partial P\left(\boldsymbol{r}_{i}, \boldsymbol{r}_{j}, t\right)}{\partial t}=D_{i} \nabla_{i} P\left(\boldsymbol{r}_{i}, \boldsymbol{r}_{j}, t\right)+D_{j} \nabla_{j} P\left(\boldsymbol{r}_{i}, \boldsymbol{r}_{j}, t\right),
$$

with a suitable boundary condition (see below). Here, $P\left(\boldsymbol{r}_{i}, \boldsymbol{r}_{j}, t\right)$ is the two-particle probability density function at time $t$. Let us change the variables as [24]

$$
\left(\begin{array}{l}
\boldsymbol{R} \\
\boldsymbol{r}
\end{array}\right)=\boldsymbol{A}\left(\begin{array}{l}
\boldsymbol{r}_{i} \\
\boldsymbol{r}_{j}
\end{array}\right) \quad \text { with } \quad \boldsymbol{A}=\left(\begin{array}{cc}
\frac{\bar{D}}{2 D_{i}} & \frac{\bar{D}}{2 D_{j}} \\
1 & -1
\end{array}\right),
$$

where $\bar{D}:=\left(D_{1}+D_{2}\right) / 2$. In each element of the matrix $\boldsymbol{A}$, the identity matrix $\boldsymbol{I}$ is omitted for brevity. After a straightforward calculation, we have [69]

$$
\frac{\partial P(\boldsymbol{R}, \boldsymbol{r}, t)}{\partial t}=D_{R} \nabla_{R} P(\boldsymbol{R}, \boldsymbol{r}, t)+D_{r} \nabla_{r} P(\boldsymbol{R}, \boldsymbol{r}, t),
$$

with a boundary condition $\partial P / \partial \boldsymbol{r}=0$ at $r=a_{i}+a_{j}$, where $D_{R}:=\bar{D}^{3} /\left(2 D_{i} D_{j}\right)$ and $D_{r}:=2 \bar{D}$. The equation (A7) can be easily separated by assuming that $P=V(\boldsymbol{R}, t) W(\boldsymbol{r}, t)$ and the boundary condition is imposed only on $W(\boldsymbol{r}, t)$. Thus, $\boldsymbol{R}$ and $\boldsymbol{r}$ defined in Eq. (A6) can be considered as mutually independent. (In Ref. [24], the fact that $\boldsymbol{R}$ and $\boldsymbol{r}$ are correlationless is proved.)

Therefore, $\boldsymbol{v}_{R}:=\partial \boldsymbol{R} / \partial t$ does not change through the collision, and only $\boldsymbol{v}_{r}:=\partial \boldsymbol{r} / \partial t$ changes just in the same way as the classical hard spheres (see Ref. [24] for a detailed discussion). To be more precise, let us define parallel and perpendicular elements as $\boldsymbol{v}=\boldsymbol{v}^{\|}+\boldsymbol{v}^{\perp}$, where $\boldsymbol{v}^{\|}:=\boldsymbol{v} \cdot \hat{\boldsymbol{r}}_{i j} \hat{\boldsymbol{r}}_{i j}$ and $\boldsymbol{v}^{\perp}:=\boldsymbol{v} \cdot\left(\boldsymbol{I}-\hat{\boldsymbol{r}}_{i j} \hat{\boldsymbol{r}}_{i j}\right)$. Then, the collision dynamics of the parallel components $\boldsymbol{v}_{R}^{\|}$and $\boldsymbol{v}_{r}^{\|}$is given by

$$
\left(\begin{array}{c}
\tilde{\boldsymbol{v}}_{R}^{\|} \\
\tilde{\boldsymbol{v}}_{r}^{\|}
\end{array}\right)=\left(\begin{array}{cc}
1 & 0 \\
0 & -1
\end{array}\right)\left(\begin{array}{c}
\boldsymbol{v}_{R}^{\|} \\
\boldsymbol{v}_{r}^{\|}
\end{array}\right),
$$

where $\tilde{\boldsymbol{v}}_{R}^{\|}$and $\tilde{\boldsymbol{v}}_{r}^{\|}$are the velocities after the collision. In contrast, the perpendicular components $\tilde{\boldsymbol{v}}_{R}^{\perp}$ and $\tilde{\boldsymbol{v}}_{r}^{\perp}$ are unchanged.

Parallel components of the post-collision pseudovelocities $\tilde{\boldsymbol{v}}_{i}$ and $\tilde{\boldsymbol{v}}_{j}$ are then given by

$$
\begin{aligned}
\left(\begin{array}{c}
\tilde{\boldsymbol{v}}_{i}^{\|} \\
\tilde{\boldsymbol{v}}_{j}^{\|}
\end{array}\right) & =\boldsymbol{A}^{-1}\left(\begin{array}{cc}
1 & 0 \\
0 & -1
\end{array}\right) \boldsymbol{A}\left(\begin{array}{c}
\boldsymbol{v}_{i}^{\|} \\
\boldsymbol{v}_{j}^{\|}
\end{array}\right) \\
& =\frac{1}{2 \bar{D}}\left(\begin{array}{cc}
D_{j}-D_{i} & 2 D_{i} \\
2 D_{j} & D_{i}-D_{j}
\end{array}\right)\left(\begin{array}{c}
\boldsymbol{v}_{i}^{\|} \\
\boldsymbol{v}_{j}^{\|}
\end{array}\right) .
\end{aligned}
$$

From these facts, the transformation of the pseudovelocities is given by

$$
\begin{aligned}
\left(\begin{array}{c}
\tilde{\boldsymbol{v}}_{i} \\
\tilde{\boldsymbol{v}}_{j}
\end{array}\right) & =\left(\begin{array}{c}
\tilde{\boldsymbol{v}}_{i}^{\|} \\
\tilde{\boldsymbol{v}}_{j}^{\|}
\end{array}\right)+\left(\begin{array}{c}
\tilde{\boldsymbol{v}}_{i}^{\perp} \\
\tilde{\boldsymbol{v}}_{j}^{\perp}
\end{array}\right) \\
& =\frac{1}{2 \bar{D}}\left(\begin{array}{cc}
D_{j}-D_{i} & 2 D_{i} \\
2 D_{j} & D_{i}-D_{j}
\end{array}\right)\left(\begin{array}{c}
\boldsymbol{v}_{i}^{\|} \\
\boldsymbol{v}_{j}^{\|}
\end{array}\right)+\left(\begin{array}{c}
\boldsymbol{v}_{i}^{\perp} \\
\boldsymbol{v}_{j}^{\perp}
\end{array}\right) \\
& =\left(\begin{array}{c}
\boldsymbol{v}_{i} \\
\boldsymbol{v}_{j}
\end{array}\right)+\frac{1}{\bar{D}}\left(\begin{array}{cc}
-D_{i} & D_{i} \\
D_{j} & -D_{j}
\end{array}\right)\left(\begin{array}{c}
v_{i}^{\|} \\
v_{j}^{\|}
\end{array}\right) \hat{\boldsymbol{r}}_{i j} .
\end{aligned}
$$

Thus, we obtain the post-collision pseudovelocities $\tilde{\boldsymbol{v}}_{i}$ and $\tilde{\boldsymbol{v}}_{j}$.

\section{APPENDIX B: NUMERICAL ESTIMATE OF LONG-TIME SELF-DIFFUSION COEFFICIENT}

The long-time diffusion coefficient displayed in Figs. 2 and 3 is estimated by using time-averaged mean square displacements (MSDs) instead of ensemble-averaged MSDs. This is because the time-averaged MSD has been frequently used in single-molecule-tracking experiments $[1-3,5,58]$ as well as molecular dynamics simulations [70,71]. The timeaveraged MSD for the particle $i$ is defined as [72-74]

$$
\overline{\delta \boldsymbol{r}_{i}^{2}}(\Delta ; T):=\frac{1}{T} \int_{0}^{T} d t^{\prime}\left[\boldsymbol{r}_{i}\left(t^{\prime}+\Delta\right)-\boldsymbol{r}_{i}\left(t^{\prime}\right)\right]^{2},
$$

where $\Delta$ is a lag time, and $T+\Delta$ is a total measurement time. The time-averaged MSD is further averaged over the particles belonging to the same species to obtain the MSD for the species $s$ as

$$
\left\langle\overline{\delta \boldsymbol{r}^{2}}(\Delta ; T)\right\rangle_{s}:=\frac{1}{n_{s}} \sum_{i=N_{s-1}+1}^{N_{s}} \overline{\delta \boldsymbol{r}_{i}^{2}}(\Delta ; T) .
$$



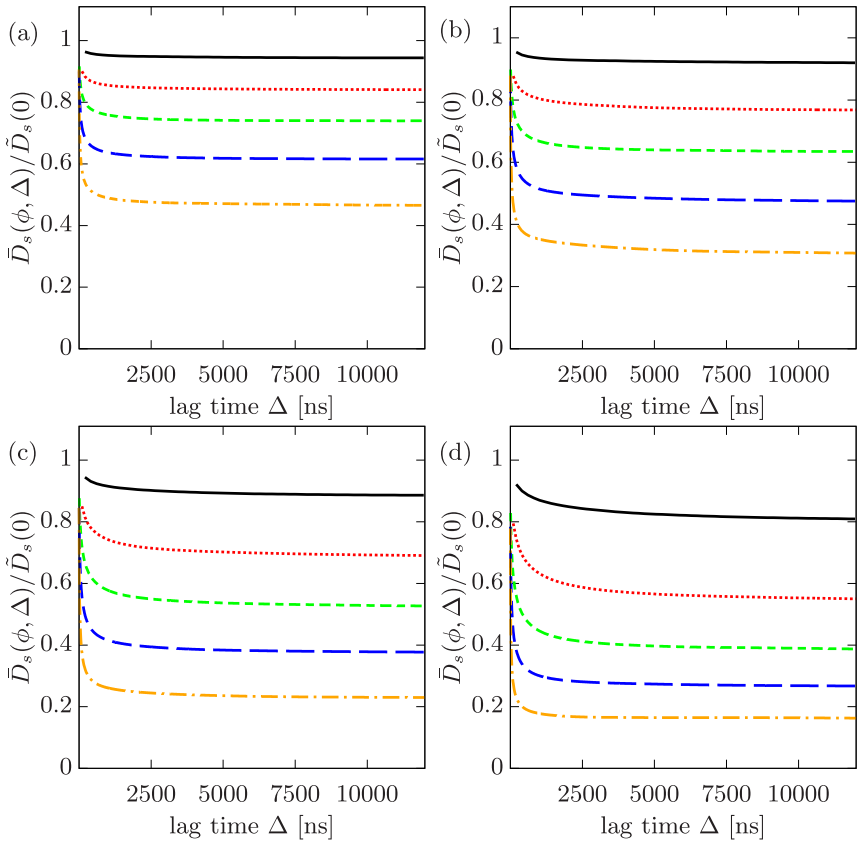

FIG. 7. Finite-time diffusion coefficient $\bar{D}_{k}(\phi, \Delta)$ [Eq. (B3)] rescaled by $D_{i}(0)$ as a function of the lag time $\Delta$ for (a) GFP, (b) glyceraldehyde-3-P dehydrogenase, (c) RNA polymerase, and (d) ribosome. In each figure, results for five different values of the volume fraction $\phi$ are displayed: $\phi=0.05$ (solid), $\phi=0.15$ (dotted), $\phi=0.25$ (dashed), $\phi=0.35$ (long-dashed), and $\phi=0.45$ (dashed-dotted).

In numerical simulations, this MSD [Eq. (B2)] is further averaged over $N_{\text {sim }}$ sets of different initial conditions $\left(N_{\text {sim }}\right.$ is set as 10 to 300 depending on the concentration $\phi$; for example, $N_{\text {sim }}=300$ for $\phi=0.025$, whereas $N_{\text {sim }}=10$ for $\phi=0.45)$.

By using the MSD, a finite-time diffusion coefficient for the species $s$ can be defined by

$$
\bar{D}_{s}(\phi, \Delta):=\frac{\left\langle\overline{\delta \boldsymbol{r}^{2}}(\Delta ; T)\right\rangle_{s}}{6 \Delta} .
$$

Then, the long-time diffusion coefficient $\bar{D}_{s}(\phi)$ for the species $s$ is given by

$$
\bar{D}_{s}(\phi):=\lim _{\Delta \rightarrow \infty} \bar{D}_{s}(\phi, \Delta) .
$$

In numerical simulations, the long-time diffusion coefficient $\bar{D}_{s}(\phi)$ is estimated for a large value of the lag time $\Delta$. In Fig. 7, the finite-time diffusion coefficient $\bar{D}_{s}(\phi, \Delta)$ is displayed for five different values of the concentration $\phi$. In each case, $\bar{D}_{s}(\phi, \Delta)$ is almost saturated to a constant at large $\Delta$, and thus the estimated value of the long-time diffusion coefficient $\bar{D}_{s}(\phi)$ should be quite accurate.

\section{APPENDIX C: REDUCTION OF DIFFUSION COEFFICIENT FOR POLYDISPERSE SUSPENSIONS WITH HI}

In this Appendix, a derivation of the long-time diffusion coefficient for the system with the HI is presented. The derivation procedure is essentially the same as that presented in Refs. [16-18]. But, here explicit formulas of the mobility coefficients $K_{s}^{\mathrm{hS}}, K_{s}^{\mathrm{hI}}$, and $K_{s}^{\mathrm{hB}}$ are given up to $O\left(1 / r^{7}\right)$. These formulas are useful in checking numerical schemes for obtaining estimates with more accuracy. For example, a numerical scheme used to obtain the data in Table I was checked by these formulas.

\section{Two-particle steady Smoluchowski equation}

In the same way as the case without the HI, we derive a steady solution $P_{12}\left(\boldsymbol{r}_{1}, \boldsymbol{r}_{2}\right)$ of a two-particle Smoluchowski equation with the HI under the condition that an external constant force $\boldsymbol{F}_{\text {ext }}$ is applied only to the particle 1. From Eqs. (1) and (24) with $N=2$, it is found that the steady solution $P_{12}\left(\boldsymbol{r}_{1}, \boldsymbol{r}_{2}\right)$ satisfies the two-particle Smoluchowski equation

$$
\begin{aligned}
0= & \nabla_{1} \cdot\left[\boldsymbol{D}_{11} \cdot\left(\beta P_{12} \nabla_{1} V_{12}-\beta P_{12} \boldsymbol{F}_{\mathrm{ext}}+\nabla_{1} P_{12}\right)\right. \\
& \left.+\boldsymbol{D}_{12} \cdot\left(\beta P_{12} \nabla_{2} V_{12}+\nabla_{2} P_{12}\right)\right] \\
& +\nabla_{2} \cdot\left[\boldsymbol{D}_{21} \cdot\left(\beta P_{12} \nabla_{1} V_{12}-\beta P_{12} \boldsymbol{F}_{\mathrm{ext}}+\nabla_{1} P_{12}\right)\right. \\
& \left.+\boldsymbol{D}_{22} \cdot\left(\beta P_{12} \nabla_{2} V_{12}+\nabla_{2} P_{12}\right)\right],
\end{aligned}
$$

where $\nabla_{1} \Phi(\boldsymbol{R})$ and $\nabla_{2} \Phi(\boldsymbol{R})$ in Eq. (24) are replaced with $\nabla_{1} V_{12}\left(r_{12}\right)-\boldsymbol{F}_{\text {ext }}$ and $\nabla_{2} V_{12}\left(r_{12}\right)$, respectively.

By using a difference vector $\boldsymbol{r}:=\boldsymbol{r}_{1}-\boldsymbol{r}_{2}$ with a gradient operator $\boldsymbol{\nabla}$ in terms of $\boldsymbol{r}$, Eq. (C1) is rewritten as

$$
\begin{aligned}
0= & \nabla \cdot\left[\left(\boldsymbol{D}_{11}-\boldsymbol{D}_{21}\right) \cdot\left(\beta P_{12} \nabla V_{12}-\beta P_{12} \boldsymbol{F}_{\mathrm{ext}}+\nabla P_{12}\right)\right. \\
& \left.+\left(\boldsymbol{D}_{22}-\boldsymbol{D}_{12}\right) \cdot\left(\beta P_{12} \nabla V_{12}+\nabla P_{12}\right)\right],
\end{aligned}
$$

where we use $\nabla=\nabla_{1}=-\nabla_{2}$. Here again, we assume that a solution $P_{12}(\boldsymbol{r})$ of Eq. (C2) takes the form of Eq. (4). Inserting Eq. (4) into Eq. (C2) and neglecting terms of $O\left(\boldsymbol{F}_{\text {ext }}^{2}\right)$, we obtain

$$
\begin{aligned}
& \boldsymbol{\nabla} \cdot\left(e ^ { - \beta V _ { 1 2 } } \left\{\left(\boldsymbol{D}_{11}-\boldsymbol{D}_{21}\right) \cdot\left[a_{12} \nabla\left(L_{12} \hat{\boldsymbol{r}} \cdot \boldsymbol{F}_{\mathrm{ext}}\right)-\boldsymbol{F}_{\mathrm{ext}}\right]\right.\right. \\
& \left.\left.\quad+\left(\boldsymbol{D}_{22}-\boldsymbol{D}_{12}\right) \cdot a_{12} \boldsymbol{\nabla}\left(L_{12} \hat{\boldsymbol{r}} \cdot \boldsymbol{F}_{\mathrm{ext}}\right)\right\}\right)=0 .
\end{aligned}
$$

Using Eqs. (25) and (28), the differences between the diffusion tensors are rewritten as

$$
\begin{aligned}
& \boldsymbol{D}_{11}-\boldsymbol{D}_{21}=D_{1}\left[B_{12} \boldsymbol{I}+\left(A_{12}-B_{12}\right) \hat{\boldsymbol{r}} \hat{\boldsymbol{r}}\right], \\
& \boldsymbol{D}_{22}-\boldsymbol{D}_{12}=D_{2}\left[B_{21} \boldsymbol{I}+\left(A_{21}-B_{21}\right) \hat{\boldsymbol{r}} \hat{\boldsymbol{r}}\right],
\end{aligned}
$$

where nondimensional functions $A_{i j}(r)$ and $B_{i j}(r)$ with $(i, j)=(1,2)$ or $(2,1)$ are defined by

$$
\begin{aligned}
& A_{i j}(r):=1+A_{i j}^{\mathrm{s}}(r)-A_{j i}^{\mathrm{c}}(r), \\
& B_{i j}(r):=1+B_{i j}^{\mathrm{s}}(r)-B_{j i}^{\mathrm{c}}(r),
\end{aligned}
$$

respectively.

Assuming that the constant external force is in the $z$ direction $\boldsymbol{F}_{\text {ext }}=\left(0,0, F_{\text {ext }}\right)$, and using $\hat{\boldsymbol{r}} \cdot \nabla=\partial / \partial r$, we obtain the following equation from Eqs. (C3)-(C7):

$$
\nabla \cdot\left[e^{-\beta V_{12}} \boldsymbol{q}_{12}(\boldsymbol{r})\right]=0,
$$


where $\boldsymbol{q}_{12}(\boldsymbol{r})$ is a three-dimensional vector given by

$$
\begin{aligned}
\boldsymbol{q}_{12}(\boldsymbol{r}):= & D_{1} B_{12}\left[a_{12} \nabla\left(L_{12} \cos \theta\right)-\hat{z}\right] \\
& +D_{1}\left(A_{12}-B_{12}\right)\left(a_{12} \frac{\partial L_{12}}{\partial r}-1\right) \hat{\boldsymbol{r}} \cos \theta \\
& +D_{2} B_{21} a_{12} \nabla\left(L_{12} \cos \theta\right) \\
& +D_{2}\left(A_{21}-B_{21}\right) a_{12} \frac{\partial L_{12}}{\partial r} \hat{\boldsymbol{r}} \cos \theta,
\end{aligned}
$$

with $\hat{z}$ being the unit vector in the direction of the $z$ axis, and $\theta$ being the angle between $\hat{\boldsymbol{r}}$ and $\hat{z}$.

Rewriting the differential operators in Eqs. (C8) and (C9) with three-dimensional polar coordinates, we obtain (after a somewhat lengthy but straightforward calculation)

$$
\begin{aligned}
& \frac{e^{-\beta V_{12}} \nabla \cdot \boldsymbol{q}_{12}(\boldsymbol{r})+\left(\nabla e^{-\beta V_{12}}\right) \cdot \boldsymbol{q}_{12}(\boldsymbol{r})}{a_{12} \cos \theta} \\
& =e^{-\beta V_{12}} \frac{J_{12}(r)}{r^{2}}+\frac{d e^{-\beta V_{12}}}{d r} \frac{K_{12}(r)}{a_{12}}=0 .
\end{aligned}
$$

Here, the function $J_{12}(r)$, which is defined by $J_{12}(r) / r^{2}:=$ $\nabla \cdot \boldsymbol{q}_{12}(\boldsymbol{r}) /\left(a_{12} \cos \theta\right)$, is expressed as

$$
\begin{aligned}
J_{12}(r)= & \left(D_{1} A_{12}+D_{2} A_{21}\right) r^{2} \frac{d^{2} L_{12}}{d r^{2}} \\
& +2\left(D_{1} B_{12}+D_{2} B_{21}\right)\left(r \frac{d L_{12}}{d r}-L_{12}\right) \\
& +\left(D_{1} w_{12}+D_{2} w_{21}\right) r \frac{d L_{12}}{d r}-D_{1} w_{12} \frac{r}{a_{12}},
\end{aligned}
$$

where a nondimensional function $w_{i j}(r)$ with $(i, j)=(1,2)$ or $(2,1)$ is defined by

$$
w_{i j}(r):=r \frac{\partial A_{i j}}{\partial r}+2\left(A_{i j}-B_{i j}\right) .
$$

Similarly, in Eq. (C10), the function $K_{12}(r)$ is defined by $K_{12}(r) / a_{12}:=\hat{\boldsymbol{r}} \cdot \boldsymbol{q}_{12}(\boldsymbol{r}) /\left(a_{12} \cos \theta\right)$, and it is expressed as

$$
K_{12}(r)=\left(D_{1} A_{12}+D_{2} A_{21}\right) a_{12} \frac{d L_{12}}{d r}-D_{1} A_{12} .
$$

By inserting Eqs. (26), (27), (29), and (30) into Eqs. (C6), (C7), and (C12), the functions $A_{12}(r), B_{12}(r)$, and $w_{12}(r)$ up to $O\left(1 / r^{7}\right)$ are obtained as

$$
\begin{aligned}
A_{12}(r)= & 1-\frac{3 a_{1}}{2 r}+\frac{a_{1}}{2 r}\left[\left(\frac{a_{1}}{r}\right)^{2}+\left(\frac{a_{2}}{r}\right)^{2}\right]-\frac{15 a_{1}}{4 r}\left(\frac{a_{2}}{r}\right)^{3} \\
& -\frac{2 a_{1}}{r}\left(\frac{a_{2}}{r}\right)^{5}+\frac{15}{2}\left(\frac{a_{1}}{r}\right)^{3}\left(\frac{a_{2}}{r}\right)^{3}-\frac{75}{4}\left(\frac{a_{1}}{r}\right)^{4}\left(\frac{a_{2}}{r}\right)^{3}, \\
B_{12}(r)= & 1-\frac{3 a_{1}}{4 r}-\frac{a_{1}}{4 r}\left[\left(\frac{a_{1}}{r}\right)^{2}+\left(\frac{a_{2}}{r}\right)^{2}\right]-\frac{17 a_{1}}{16 r}\left(\frac{a_{2}}{r}\right)^{5}, \\
w_{12}(r)= & \frac{15 a_{1}}{2 r}\left(\frac{a_{2}}{r}\right)^{3}+\frac{81 a_{1}}{8 r}\left(\frac{a_{2}}{r}\right)^{5} \\
& -30\left(\frac{a_{1}}{r}\right)^{3}\left(\frac{a_{2}}{r}\right)^{3}+\frac{375}{4}\left(\frac{a_{1}}{r}\right)^{4}\left(\frac{a_{2}}{r}\right)^{3} .
\end{aligned}
$$

Moreover, $A_{21}(r), B_{21}(r)$, and $w_{21}(r)$ are obtained by exchanging the subscripts 1 and 2 .

\section{Calculation of $L_{12}(r)$}

To derive a far-field approximation for the steady solution, we expand the function $L_{12}(r)$ in Eq. (4) in terms of the inverse distance as [15]

$$
L_{12}(r)=\sum_{n=1}^{M-1}\left(\frac{a_{12}}{r}\right)^{n} \alpha_{n}+O\left(\left(\frac{a_{12}}{r}\right)^{M}\right),
$$

where $M$ is the order of the far-field approximation, and for explicit formulas given below it is set as $M=7$. Moreover, the nondimensional parameters $\alpha_{n}$ should be written as $\alpha_{12, n}$ because it depends on the particle indices (here, 1 and 2); but, for the sake of brevity, we use the simple notation $\alpha_{n}$.

For the hard-sphere interaction, $V_{12}(r)=0$, if $r>a_{1}+a_{2}$. Thus, from Eq. (C10), we have $J_{12}(r) \equiv 0$ for $r>a_{1}+a_{2}$. Therefore, when substituting Eq. (C15) into Eq. (C11), each coefficient of $1 / r^{n}(n=1, \ldots, 6)$ must be vanishing. Thus, we have the following set of formulas:

$$
\begin{gathered}
\alpha_{1}=0, \\
\alpha_{3}=\frac{3}{8}\left(3 b^{11} \alpha_{2}+\frac{5 b^{14}}{2}\right), \\
\alpha_{4}=\frac{9}{20}\left(3 b^{22} \alpha_{2}+\frac{5 b^{25}}{2}\right), \\
\alpha_{5}=\frac{1}{4}\left(\frac{27 b^{33}}{4}-b^{13}-b^{31}\right) \alpha_{2}+\frac{45 b^{36}}{32}-\frac{5 b^{34}}{6}+\frac{9 b^{16}}{32}, \\
\alpha_{6}=\frac{3}{28}\left[\frac{81}{4} b^{44}-6\left(b^{24}+b^{42}\right)+\frac{25}{4}\left(b^{14}+b^{41}\right)\right] \alpha_{2} \\
+\frac{15}{56}\left(\frac{27 b^{47}}{4}-5 b_{45}+\frac{7 b^{27}}{20}+\frac{25 b^{44}}{4}\right), \quad(\mathrm{C} 20)
\end{gathered}
$$

where, to express these formulas with $a_{i}$ instead of $D_{i}$, the Stokes-Einstein relation $D_{i}=k_{B} T /\left(6 \pi \eta a_{i}\right)(i=1,2)$ is used. Moreover, $b^{\alpha \beta}$ is defined as

$$
b^{\alpha \beta}:=\frac{a_{1}^{\alpha} a_{2}^{\beta}}{a_{12}^{\alpha+\beta}} .
$$

Note that, if the value of $\alpha_{2}$ is obtained, $\alpha_{3}, \ldots, \alpha_{6}$ can be calculated by using Eqs. (C16)-(C20).

The value of $\alpha_{2}$ can be determined by a boundary condition as follows. Since $d e^{-\beta V_{12}(r)} / d r=\delta\left(r-2 a_{12}\right)$ for the hardsphere interaction, integrating Eq. (C10) over an infinitesimal interval $\left(2 a_{12}-\epsilon, 2 a_{12}+\epsilon\right)$, we obtain

$$
K_{12}\left(2 a_{12}\right)=0
$$

Evaluating Eq. (C22) with Eqs. (C16)-(C20), we have the following formula for $\alpha_{2}$ :

$$
\alpha_{2}=-\frac{1}{Q_{12}}\left[R_{12}+\frac{2^{8}}{1+\frac{a_{1} A_{21}\left(2 a_{21}\right)}{a_{2} A_{12}\left(2 a_{12}\right)}}\right],
$$


where $Q_{12}$ and $R_{12}$ are functions of the particles' radii $a_{1}$ and $a_{2}$, and defined, respectively, by

$$
\begin{aligned}
Q_{12}:= & 64+54 b^{11}+\frac{216}{5} b^{22}+\frac{135}{4} b^{33}+\frac{729}{28} b^{44} \\
& -\left(b^{13}+b^{31}\right)\left(5+\frac{54}{7} b^{11}\right)+\frac{225}{28}\left(b^{14}+b^{41}\right), \\
R_{12}:= & 9\left[b^{14}\left(5+4 b^{11}+\frac{25}{8} b^{22}+\frac{135}{56} b^{33}\right)\right. \\
& \left.+b^{16}\left(\frac{5}{8}+\frac{1}{8} b^{11}\right)-b^{34}\left(\frac{50}{27}+\frac{25}{14} b^{11}\right)+\frac{125}{56} b^{44}\right] .
\end{aligned}
$$

For given values of $a_{1}$ and $a_{2}$, the coefficients $\alpha_{j}(j=$ $1, \ldots, 6)$ can be calculated by means of Eqs. (C16)-(C24). Then, a far-field approximation of the steady solution is given by Eq. (4) with Eq. (C15).

By using these values of $\alpha_{j}(j=1, \ldots, 6)$ in Eq. (C15), we obtain the function $L_{12}(r)$ up to $O\left(1 / r^{6}\right)$. Here, the subscripts 1 and 2 of $L_{12}(r)$ indicate the particle indices, whereas we also use a notation $\tilde{L}_{s k}(r)$ to refer to the same function but for the species $s$ and $k$. To obtain explicit form of $\tilde{L}_{s k}(r)$, just replace $a_{1}, a_{2}$, and $a_{12}$ in the expansion of $L_{12}(r)$ with $\tilde{a}_{s}, \tilde{a}_{k}$, and $\tilde{a}_{s k}$, respectively.

\section{Short-time self-diffusion}

In this section, the short-time diffusion coefficient is investigated. The short-time diffusion coefficient for the system without the $\mathrm{HI}$ is equivalent to that at infinite dilution $D_{i}(0)$ because particles do not interact with each other for timescales shorter than a mean collision time. In contrast, for the system with the HI, the short-time diffusion coefficient is different from $D_{i}(0)$ because the particles interact with each other through the $\mathrm{HI}$ even for the short-time scale.

Let us denote a short-time diffusion coefficient of the particle $i$ as $D_{i}^{\mathrm{hS}}(\phi)$. The short-time diffusion coefficient for isotropic systems is simply given by

$$
D_{i}^{\mathrm{hS}}(\phi) \boldsymbol{I}=\left\langle\boldsymbol{D}_{i i}\right\rangle_{\mathrm{eq}},
$$

where $\langle\ldots\rangle_{\text {eq }}$ is the equilibrium average (see Chap. 6 in Ref. [15] for a derivation). To make the notation simple, we set $i=1$ hereafter; note that the particle 1 belongs to the first species $s=1$. From Eq. (25), we have

$$
\begin{aligned}
\left\langle\boldsymbol{D}_{11}\right\rangle_{\mathrm{eq}}= & D_{1} \boldsymbol{I}+D_{1} \sum_{l=2}^{N}\left\langle A_{1 l}^{\mathrm{s}}\left(r_{1 l}\right) \hat{\boldsymbol{r}}_{1 l} \hat{\boldsymbol{r}}_{1 l}+B_{1 l}^{\mathrm{s}}\left(r_{1 l}\right)\left(\boldsymbol{I}-\hat{\boldsymbol{r}}_{1 l} \hat{\boldsymbol{r}}_{1 l}\right)\right\rangle_{\mathrm{eq}} \\
\approx & D_{1} \boldsymbol{I}+D_{1} \sum_{k=1}^{S} n_{k}\left(\tilde{A}_{1 k}^{\mathrm{s}}\left(r_{1 N_{k}}\right) \hat{\boldsymbol{r}}_{1 N_{k}} \hat{\boldsymbol{r}}_{1 N_{k}}\right. \\
& \left.+\mathrm{B}_{1 k}^{\mathrm{s}}\left(r_{1 N_{k}}\right)\left(\boldsymbol{I}-\hat{\boldsymbol{r}}_{1 N_{k}} \hat{\boldsymbol{r}}_{1 N_{k}}\right)\right\rangle_{\mathrm{eq}},
\end{aligned}
$$

where we use the fact that the ensemble averages for the $n_{k}$ particles belonging to the species $k$ are equivalent. Moreover, the approximation $n_{1}-1 \approx n_{1}$ is used in the second equality.

In the ensemble average in Eq. (C26), integrals should be taken over the supervector $\boldsymbol{R}=\left(\boldsymbol{r}_{1}, \ldots, \boldsymbol{r}_{N}\right)$ in terms of the equilibrium $N$-particle distribution $P^{\mathrm{eq}}(\boldsymbol{R})$. But, the integrals over $\boldsymbol{r}_{j}$ with $j \neq 1, N_{k}$ can be readily carried out, whereas the integrals over $\boldsymbol{r}_{1}$ and $\boldsymbol{r}_{N_{k}}$ are expressed in terms of spherical coordinates. Accordingly, the ensemble average in Eq. (C26) is rewritten as

$$
\boldsymbol{I} \frac{4 \pi}{3 V} \int_{0}^{\infty} d r g_{1 N_{k}}^{\mathrm{eq}}(r) r^{2}\left[\tilde{A}_{1 k}^{\mathrm{s}}(r)+2 \tilde{B}_{1 k}^{\mathrm{s}}(r)\right],
$$

where $g_{i j}^{\mathrm{eq}}(r)$ is the equilibrium pair correlation function of the particles $i$ and $j$, and $\boldsymbol{r}$ is defined as $\boldsymbol{r}:=\boldsymbol{r}_{1 N_{k}}$. To the leading order in the volume fraction, $g_{i j}^{\mathrm{eq}}(r)$ can be approximated as $g_{i j}^{\mathrm{eq}}(r) \approx \exp \left[-\beta V_{i j}(r)\right]$ (see Chap. 1 in Ref. [15]). For the hard-sphere systems, $g_{i j}^{\mathrm{eq}}(r)=1$ for $r>2 a_{i j}$, otherwise $g_{i j}^{\mathrm{eq}}(r)=0$. Thus, by changing the variables as $r=\tilde{a}_{1 k} x$ and using Eqs. (26) and (27), Eq. (C27) can be rewritten as

$$
\begin{array}{r}
\boldsymbol{I} \frac{4 \pi \tilde{a}_{1 k}^{3}}{3 V} \int_{2}^{\infty} d x x^{2}\left[\tilde{A}_{1 k}^{\mathrm{s}}\left(\tilde{a}_{1 k} x\right)+2 \tilde{B}_{1 k}^{\mathrm{s}}\left(\tilde{a}_{1 k} x\right)\right] \\
\quad=\boldsymbol{I} \frac{4 \pi \tilde{a}_{k}^{3}}{3 V} \frac{\tilde{a}_{1}}{\tilde{a}_{1 k}}\left[-\frac{15}{8}-\frac{11}{64} \frac{\tilde{a}_{k}^{2}}{\tilde{a}_{1 k}^{2}}+\frac{5}{16} \frac{\tilde{a}_{1}^{2}}{\tilde{a}_{1 k}^{2}}\right],
\end{array}
$$

where $\tilde{a}_{s}$ is the radius of species $s$, and $\tilde{a}_{k s}=\left(\tilde{a}_{k}+\tilde{a}_{s}\right) / 2$ as in Sec. II C. Comparing Eq. (C25) with Eqs. (C26)-(C28), we have

$$
D_{1}^{\mathrm{hS}}(\phi)=D_{1}\left(1-K_{1}^{\mathrm{hS}} \phi\right),
$$

where $K_{s}^{\mathrm{hS}}(s=1, \ldots, S)$ is a mobility coefficient for the species $s$ and is given by

$$
K_{s}^{\mathrm{hS}}=\frac{\sum_{k=1}^{S} n_{k} \tilde{a}_{k}^{3} \tilde{a}_{s}\left(\frac{15}{8}+\frac{11}{64} \frac{\tilde{a}_{k}^{2}}{\tilde{a}_{s k}}-\frac{5}{16} \frac{\tilde{a}_{s}^{2}}{\tilde{a}_{s k}^{2}}\right)}{\sum_{k=1}^{S} n_{k} \tilde{a}_{k}^{3}} .
$$

Note that $K_{s}^{\mathrm{hS}}$ is independent of the volume fraction $\phi$. If we denote the short-time diffusion coefficients for the species $s$ as $\tilde{D}_{s}^{\text {hS }}(\phi)$, Eq. (C29) is rewritten as

$$
\tilde{D}_{s}^{\mathrm{hS}}(\phi)=\tilde{D}_{s}(0)\left(1-K_{s}^{\mathrm{hS}} \phi\right) \quad(s=1, \ldots, S) .
$$

\section{Long-time self-diffusion}

Here, we derive the long-time self-diffusion coefficient up to $O(\phi)$. Suppose that the constant external force $\boldsymbol{F}^{\mathrm{ext}}$ is exerted on the particle 1 . Then, the drift velocity $\left\langle\boldsymbol{v}_{1}\right\rangle$ of that particle is given by

$$
\left\langle\boldsymbol{v}_{1}\right\rangle=\beta \sum_{j=1}^{N}\left\langle\boldsymbol{D}_{1 j} \cdot\left[\boldsymbol{F}_{\mathrm{ext}} \delta_{1 j}-\nabla_{j} \Phi(\boldsymbol{R})-k_{B} T \nabla_{j} \ln P\right]\right\rangle,
$$

where the inside of the square brackets is the force exerted on the particle $j$, and the ensemble average $\langle\ldots\rangle$ should be taken with respect to the steady state, which is nonequilibrium due to the external force $\boldsymbol{F}^{\text {ext }}$. By using Eqs. (C25) and (C29), the above equation can be rewritten as

$$
\left\langle\boldsymbol{v}_{1}\right\rangle \approx \beta D_{1}\left(1-K_{1}^{\mathrm{hS}} \phi\right) \boldsymbol{F}_{\mathrm{ext}}+\left\langle\boldsymbol{v}_{1}^{\mathrm{I}}\right\rangle+\left\langle\boldsymbol{v}_{1}^{\mathrm{B}}\right\rangle,
$$

where, in deriving the first term, we neglected higher-order terms with respect to $\boldsymbol{F}_{\text {ext }}$ by using the approximation $\langle\ldots\rangle$. $\boldsymbol{F}_{\mathrm{ext}} \simeq\langle\ldots\rangle_{\mathrm{eq}} \cdot \boldsymbol{F}_{\mathrm{ext}}$. In addition, $\left\langle\boldsymbol{v}_{1}^{\mathrm{I}}\right\rangle$ and $\left\langle\boldsymbol{v}_{1}^{\mathrm{B}}\right\rangle$ are the drift 
velocities due to the direct interaction and the Brownian force, and they are given by

$$
\begin{aligned}
\left\langle\boldsymbol{v}_{1}^{\mathrm{I}}\right\rangle & =-\beta \sum_{j=1}^{N}\left\langle\boldsymbol{D}_{1 j} \cdot \nabla_{j} \Phi(\boldsymbol{R})\right\rangle \\
& \approx-\beta\left\langle\boldsymbol{D}_{11} \cdot \nabla_{1} \Phi\right\rangle-\beta \sum_{k=1}^{S} n_{k}\left\langle\boldsymbol{D}_{1 N_{k}} \cdot \nabla_{N_{k}} \Phi\right\rangle \\
\left\langle\boldsymbol{v}_{1}^{\mathrm{B}}\right\rangle & =-\sum_{j=1}^{N}\left\langle\boldsymbol{D}_{1 j} \cdot \nabla_{j} \ln P(\boldsymbol{R})\right\rangle \\
& \approx-\left\langle\boldsymbol{D}_{11} \cdot \nabla_{1} \ln P\right\rangle-\sum_{k=1}^{S} n_{k}\left\langle\boldsymbol{D}_{1 N_{k}} \cdot \nabla_{N_{k}} \ln P\right\rangle
\end{aligned}
$$

where the approximation $n_{1}-1 \approx n_{1}$ is used.

\section{a. Direct interaction}

First, let us calculate the velocity $\left\langle\boldsymbol{v}_{1}^{\mathrm{I}}\right\rangle$ by assuming that the potential energy is given by a sum of pair potentials as $\Phi(\boldsymbol{R})=\sum_{i, j=1, i<j}^{N} V_{i j}\left(r_{i j}\right)$. By using $V_{i j}(r)=V_{j i}(r)$ and $r_{i j}=r_{j i}$, we have $\nabla_{l} \Phi(\boldsymbol{R})=\sum_{j=1, j \neq l}^{N} \nabla_{l} V_{j l}\left(r_{j l}\right)$, and thus Eq. (C34) becomes

$$
\begin{aligned}
\left\langle\boldsymbol{v}_{1}^{\mathrm{I}}\right\rangle \approx-\beta \sum_{k=1}^{S} n_{k}\left[\left\langle\boldsymbol{D}_{11} \cdot \nabla_{1} V_{1 N_{k}}\left(r_{1 N_{k}}\right)\right\rangle\right. & \\
& \left.+\sum_{\substack{j=1 \\
j \neq N_{k}}}^{N}\left\langle\boldsymbol{D}_{1 N_{k}} \cdot \nabla_{N_{k}} V_{j N_{k}}\left(r_{j N_{k}}\right)\right\rangle\right] .
\end{aligned}
$$

By substituting Eq. (25) into the first term in the right side of Eq. (C36), a summation $\sum_{l=2}^{N}$ appears. But, the terms with $l \neq N_{k}$ are of $O\left(\phi^{2}\right)$ and can be neglected. This is because a three-particle correlation function $g^{(3)}\left(\boldsymbol{r}_{1}, \boldsymbol{r}_{l}, \boldsymbol{r}_{N_{k}}\right) / V^{3}$ encountered in the ensemble average can be approximated as $g^{(3)}\left(\boldsymbol{r}_{1}, \boldsymbol{r}_{l}, \boldsymbol{r}_{N_{k}}\right) / V^{3} \approx g_{1 l}\left(\boldsymbol{r}_{1 l}\right) g_{1 N_{k}}\left(\boldsymbol{r}_{1 N_{k}}\right) g_{l N_{k}}\left(\boldsymbol{r}_{l N_{k}}\right) / V^{3}$ for the leading order in $\phi$. Integration over one of the three variables is readily carried out, and results in $V$; the remaining double integral is independent of $V$. Hence, the terms with $l \neq N_{k}$ are proportional to $1 / V^{2}$, and thus they are of $O\left(\phi^{2}\right)$. Similarly, Eq. (28) is substituted into the second term in Eq. (C36). Then, again, the terms with $j \neq 1$ are of higher order in $\phi$, and thus we also neglect these terms.

Consequently, we have

$$
\begin{aligned}
\left\langle\boldsymbol{v}_{1}^{\mathrm{I}}\right\rangle \approx & -\beta D_{1} \sum_{k=1}^{S} n_{k}\left\langle\left[ G_{1 k}\left(r_{1 N_{k}}\right) \hat{\boldsymbol{r}}_{1 N_{k}} \hat{\boldsymbol{r}}_{1 N_{k}}\right.\right. \\
& \left.\left.+H_{1 k}\left(r_{1 N_{k}}\right)\left(\boldsymbol{I}-\hat{\boldsymbol{r}}_{1 N_{k}} \hat{\boldsymbol{r}}_{1 N_{k}}\right)\right] \cdot \nabla_{1} V_{1 N_{k}}\left(r_{1 N_{k}}\right)\right\rangle \\
= & -\beta D_{1} \sum_{k=1}^{S} n_{k} V \int d \boldsymbol{r} P_{1 N_{k}}(\boldsymbol{r})\left[G_{1 k}(r) \hat{\boldsymbol{r}} \hat{\boldsymbol{r}}\right. \\
& \left.+H_{1 k}(r)(\boldsymbol{I}-\hat{\boldsymbol{r}} \hat{\boldsymbol{r}})\right] \cdot \hat{\boldsymbol{r}} \frac{\partial V_{1 N_{k}}(r)}{\partial r},
\end{aligned}
$$

where $\nabla_{N_{k}} f\left(r_{1 N_{k}}\right)=-\nabla_{1} f\left(r_{1 N_{k}}\right)$ is used. The functions $G_{s k}(r)$ and $H_{s k}(r)$ are defined, respectively, as [19]

$$
\begin{aligned}
G_{s k}(r) & :=1+\tilde{A}_{s k}^{\mathrm{s}}(r)-\frac{\tilde{a}_{s}}{\tilde{a}_{k}} \tilde{A}_{s k}^{\mathrm{c}}(r), \\
H_{s k}(r) & :=1+\tilde{B}_{s k}^{\mathrm{s}}(r)-\frac{\tilde{a}_{s}}{\tilde{a}_{k}} \tilde{B}_{s k}^{\mathrm{c}}(r) .
\end{aligned}
$$

Moreover, for obtaining Eq. (C38), we set $\boldsymbol{r}_{1 N_{k}}:=\boldsymbol{r}$ and integrated over the remaining $N-1$ variables. Note also that the term with $H_{1 k}(r)$ in Eq. (C38) vanishes because $(\boldsymbol{I}-\hat{\boldsymbol{r}} \hat{\boldsymbol{r}})$. $\hat{\boldsymbol{r}}=0$.

Now, let us substitute Eq. (4) into $P_{1 N_{k}}(\boldsymbol{r})$ in Eq. (C38). Namely, we set $P_{1 N_{k}}(\boldsymbol{r})=e^{-\beta V_{1 N_{k}}(r)} / V^{2}\left[1+\beta \tilde{a}_{1 k} \tilde{L}_{1 k}(r) \hat{\boldsymbol{r}}\right.$. $\left.\boldsymbol{F}_{\text {ext }}\right]$, where the function $\tilde{L}_{1 k}(r)$ is derived in Appendix C 2, but, here, indices 1 and $k$ should be considered as the species indices, and thus $a_{1}, a_{2}$, and $a_{12}$ appeared in Eqs. (C15)-(C20) should be replaced by $\tilde{a}_{1}, \tilde{a}_{k}$, and $\tilde{a}_{1 k}$. Then, the integral in Eq. (C38) can be carried out, by using $-\beta e^{-\beta V_{1 N_{k}}(r)} \partial V_{1 N_{k}}(r) / \partial r=\delta\left(r-2 \tilde{a}_{1 k}\right)$ as well as $\int d \Omega \hat{\boldsymbol{r}}=0$ and $\int d \Omega \hat{\boldsymbol{r}} \hat{\boldsymbol{r}}=4 \pi \boldsymbol{I} / 3$. Thus, we obtain the drift velocity due to the hard-sphere interaction as

$$
\left\langle\boldsymbol{v}_{1}^{\mathrm{I}}\right\rangle \approx-\beta D_{1} K_{1}^{\mathrm{hI}} \phi \boldsymbol{F}_{\mathrm{ext}},
$$

where $K_{s}^{\mathrm{hI}}$ is a mobility coefficient for the species $s$, and it is given by

$$
K_{s}^{\mathrm{hI}}=-\frac{4 \sum_{k=1}^{S} n_{k} \tilde{a}_{s k}^{3} \tilde{L}_{s k}\left(2 \tilde{a}_{s k}\right) G_{s k}\left(2 \tilde{a}_{s k}\right)}{\sum_{k=1}^{S} n_{k} \tilde{a}_{k}^{3}}
$$

for $s=1, \ldots, S$. The value of $K_{s}^{\mathrm{hI}}$ calculated by Eq. (C42) is quite small. This is consistent with the analysis of a resistance matrix [22], where it is shown that $G_{s k}\left(2 \tilde{a}_{s k}\right)=0$ in general. Thus, there is no contribution from the direct interaction, i.e., $K_{s}^{\mathrm{hI}}=0$ for the hard-sphere interaction.

\section{b. Brownian force}

Next, the drift velocity due to the Brownian force $\left\langle\boldsymbol{v}_{1}^{\mathrm{hB}}\right\rangle$ is calculated. First, the $N$-point probability density function $P(\boldsymbol{R})$ is approximated as $P(\boldsymbol{R}) \propto g^{N}(\boldsymbol{R}) \simeq \prod_{i<j}^{N} g_{i j}\left(\boldsymbol{r}_{i j}\right)$, where $g^{N}(\boldsymbol{R})$ is the $N$-point correlation function. Thus, we have $\nabla_{l} \ln P(\boldsymbol{R}) \simeq \sum_{j=1, j \neq l}^{N} \nabla_{l} \ln g_{l j}\left(\boldsymbol{r}_{l j}\right)$, and thus Eq. (C35) becomes

$$
\begin{aligned}
\left\langle\boldsymbol{v}_{1}^{\mathrm{B}}\right\rangle \approx-\sum_{k=1}^{S} n_{k} & {\left[\boldsymbol{D}_{11} \cdot \nabla_{1} g_{1 N_{k}}\left(\boldsymbol{r}_{1 N_{k}}\right)\right\rangle } \\
& \left.+\sum_{\substack{j=1 \\
j \neq N_{k}}}^{N}\left\langle\boldsymbol{D}_{1 N_{k}} \cdot \nabla_{N_{k}} g_{j N_{k}}\left(\boldsymbol{r}_{j N_{k}}\right)\right\rangle\right] .
\end{aligned}
$$

Let us substitute Eq. (25) into the first term on the right side of Eq. (C43). Then, in the same way as the case of $\left\langle\boldsymbol{v}_{1}^{\mathrm{hI}}\right\rangle$, we can neglect the terms with $l \neq N_{k}$ because they are higher order in concentration. Similarly, in the second term on the right side of Eq. (C43), the terms with $j \neq 1$ can be neglected. 
Consequently, we obtain

$$
\begin{aligned}
\left\langle\boldsymbol{v}_{1}^{B}\right\rangle \approx & -D_{1} \sum_{k=1}^{S} n_{k}\left\langle\left[ G_{1 k}\left(r_{1 N_{k}}\right) \hat{\boldsymbol{r}}_{1 N_{k}} \hat{\boldsymbol{r}}_{1 N_{k}}\right.\right. \\
& \left.\left.+H_{1 k}\left(r_{1 N_{k}}\right)\left(\boldsymbol{I}-\hat{\boldsymbol{r}}_{1 N_{k}} \hat{\boldsymbol{r}}_{1 N_{k}}\right)\right] \cdot \nabla_{1} \ln g_{1 N_{k}}\left(\boldsymbol{r}_{1 N_{k}}\right)\right\rangle(\mathrm{C} 44) \\
= & -D_{1} \sum_{k=1}^{S} \frac{n_{k}}{V} \int d \boldsymbol{r}\left[G_{1 k}(r) \hat{\boldsymbol{r}} \hat{\boldsymbol{r}}\right. \\
& \left.+H_{1 k}(r)(\boldsymbol{I}-\hat{\boldsymbol{r}} \hat{\boldsymbol{r}})\right] \cdot \nabla g_{1 N_{k}}(\boldsymbol{r}) .
\end{aligned}
$$

By using Eq. (4) with $P_{1 N_{k}}(r)=g_{1 N_{k}}(r) / V^{2}, \nabla g_{1 N_{k}}(\boldsymbol{r})$ can be expressed as

$$
\begin{aligned}
\nabla g_{1 N_{k}}(\boldsymbol{r})= & \hat{\boldsymbol{r}} \frac{\partial e^{-\beta V_{1 N_{k}}(r)}}{\partial r}+\beta \tilde{a}_{1 k}\left[\frac{\partial e^{-\beta V_{1 N_{k}}(r)} \tilde{L}_{1 k}(r)}{\partial r} \hat{\boldsymbol{r}} \hat{\boldsymbol{r}}\right. \\
& \left.+\frac{e^{-\beta V_{1 N_{k}}(r)} \tilde{L}_{1 k}(r)}{r}(\boldsymbol{I}-\hat{\boldsymbol{r}} \hat{\boldsymbol{r}})\right] \cdot \boldsymbol{F}_{\mathrm{ext}} .
\end{aligned}
$$

Here, the first term in Eq. (C46) does not contribute to the integration in Eq. (C45) because $\int d \Omega \hat{\boldsymbol{r}}=0$. Substituting Eq. (C46) into Eq. (C45), we obtain

$$
\left\langle\boldsymbol{v}_{1}^{\mathrm{hB}}\right\rangle \approx-\beta D_{1} K_{1}^{\mathrm{hB}} \phi \boldsymbol{F}_{\mathrm{ext}},
$$

where $K_{s}^{\mathrm{hB}}$ is a mobility coefficient for the species $s . K_{s}^{\mathrm{hB}}$ is given by

$$
K_{s}^{\mathrm{hB}}=-\frac{\sum_{k=1}^{S} n_{k} \tilde{a}_{s k}^{3} \int_{2}^{\infty} d x x \tilde{L}_{s k}\left(\tilde{a}_{s k} x\right) W_{s k}\left(\tilde{a}_{s k} x\right)}{\sum_{k=1}^{S} n_{k} \tilde{a}_{k}^{3}}
$$

for $s=1, \ldots, S$. Here, the function $W_{s k}(r)$ is defined as

$$
\begin{aligned}
W_{s k}(r) & :=r \frac{\partial G_{s k}(r)}{\partial r}+2\left[G_{s k}(r)-H_{s k}(r)\right] \\
& =\frac{15 \tilde{a}_{s} \tilde{a}_{k}^{3}}{2 r^{4}}+\frac{81 \tilde{a}_{s} \tilde{a}_{k}^{5}}{8 r^{6}}-30 \frac{\tilde{a}_{s}^{3} \tilde{a}_{k}^{3}}{r^{6}}+\frac{375 \tilde{a}_{s}^{4} \tilde{a}_{k}^{3}}{4 r^{7}} .
\end{aligned}
$$

Substituting Eqs. (C15) and (C49) into Eq. (C48) and then carrying out the integration, we obtain an explicit form of the mobility coefficient $K_{s}^{\mathrm{hB}}$; an explicit expression for the integral of Eq. (C48) is expressed as

$$
\begin{gathered}
\sum_{n=2}^{6} \frac{3 \alpha_{n}}{2^{n+7}}\left[\frac{80}{n+2} \frac{\tilde{a}_{s} \tilde{a}_{k}^{3}}{\tilde{a}_{s k}^{4}}+\frac{27}{n+4} \frac{\tilde{a}_{s} \tilde{a}_{k}^{5}}{\tilde{a}_{s k}^{6}}\right. \\
\left.-\frac{80}{n+4} \frac{\tilde{a}_{s}^{3} \tilde{a}_{k}^{3}}{\tilde{a}_{s k}^{6}}+\frac{125}{n+5} \frac{\tilde{a}_{s}^{4} \tilde{a}_{k}^{3}}{\tilde{a}_{s k}^{7}}\right] .
\end{gathered}
$$

\section{c. Reduction of diffusion coefficient}

Finally, here let us derive the diffusion coefficient as a function of the concentration $\phi$. From Eqs. (C33), (C41), and (C47), we obtain

$$
\begin{aligned}
\left\langle\boldsymbol{v}_{1}\right\rangle & \approx \beta D_{1}\left[1-\left(K_{1}^{\mathrm{hS}}+K_{1}^{\mathrm{hI}}+K_{1}^{\mathrm{hB}}\right) \phi\right] \boldsymbol{F}_{\mathrm{ext}} \\
& =\beta D_{1}\left(1-K_{1}^{\mathrm{h}} \phi\right) \boldsymbol{F}_{\mathrm{ext}},
\end{aligned}
$$

where $K_{s}^{\mathrm{h}}$ is the total mobility coefficient for the species $s$ defined in Eq. (33). Using the Einstein relation and Eq. (C51) just as in the case without the HI [see the derivation of Eq. (18)], the diffusion coefficient is given by $D_{1}(\phi)=$ $D_{1}(0)\left(1-K_{1}^{\mathrm{h}} \phi\right)$; or, more generally Eq. (32) holds for the species $s(s=1, \ldots, S)$.
[1] I. Golding and E. C. Cox, Phys. Rev. Lett. 96, 098102 (2006).

[2] J.-H. Jeon, V. Tejedor, S. Burov, E. Barkai, C. Selhuber-Unkel, K. Berg-Sørensen, L. Oddershede, and R. Metzler, Phys. Rev. Lett. 106, 048103 (2011).

[3] S. M. A. Tabei, S. Burov, H. Y. Kim, A. Kuznetsov, T. Huynh, J. Jureller, L. H. Philipson, A. R. Dinner, and N. F. Scherer, Proc. Natl. Acad. Sci. USA 110, 4911 (2013).

[4] J. A. Dix and A. Verkman, Annu. Rev. Biophys. 37, 247 (2008).

[5] B. R. Parry, I. V. Surovtsev, M. T. Cabeen, C. S. O'Hern, E. R. Dufresne, and C. Jacobs-Wagner, Cell 156, 183 (2014).

[6] O. G. Berg, R. B. Winter, and P. H. Von Hippel, Biochemistry 20, 6929 (1981)

[7] M. B. Elowitz, M. G. Surette, P.-E. Wolf, J. B. Stock, and S. Leibler, J. Bacteriol. 181, 197 (1999).

[8] M. C. Konopka, I. A. Shkel, S. Cayley, M. T. Record, and J. C. Weisshaar, J. Bacteriol. 188, 6115 (2006).

[9] S. B. Zimmerman and S. O. Trach, J. Mol. Biol. 222, 599 (1991).

[10] D. Ridgway, G. Broderick, A. Lopez-Campistrous, M. Ru'aini, P. Winter, M. Hamilton, P. Boulanger, A. Kovalenko, and M. J. Ellison, Biophys. J. 94, 3748 (2008).

[11] T. Ando and J. Skolnick, Proc. Natl. Acad. Sci. USA 107, 18457 (2010).
[12] J. Schöneberg and F. Noé, PloS One 8, e74261 (2013).

[13] S. Hasnain, C. L. McClendon, M. T. Hsu, M. P. Jacobson, and P. Bandyopadhyay, PLoS One 9, e106466 (2014).

[14] S. Kim and S. J. Karrila, Microhydrodynamics: Principles and Selected Applications (Dover, New York, 2013).

[15] J. K. G. Dhont, An Introduction to Dynamics of Colloids (Elsevier, Amsterdam, 1996).

[16] G. K. Batchelor, J. Fluid Mech. 119, 379 (1982).

[17] G. K. Batchelor, J. Fluid Mech. 131, 155 (1983).

[18] G. K. Batchelor, J. Fluid Mech. 137, 467 (1983).

[19] B. Cichocki and B. U. Felderhof, J. Chem. Phys. 89, 3705 (1988).

[20] B. Cichocki and B. U. Felderhof, J. Chem. Phys. 89, 1049 (1988).

[21] H. N. W. Lekkerkerker and J. K. G. Dhont, J. Chem. Phys. 80, 5790 (1984).

[22] D. J. Jeffrey and Y. Onishi, J. Fluid Mech. 139, 261 (1984).

[23] A. Scala, T. Voigtmann, and C. De Michele, J. Chem. Phys. 126, 134109 (2007).

[24] A. Scala, Eur. Phys. J.: Spec. Top. 216, 21 (2013).

[25] J. P. Bouchaud, J. Phys. I (France) 2, 1705 (1992).

[26] M. J. Saxton, Biophys. J. 66, 394 (1994).

[27] D. S. Banks and C. Fradin, Biophys. J. 89, 2960 (2005). 
[28] Y. He, S. Burov, R. Metzler, and E. Barkai, Phys. Rev. Lett. 101, 058101 (2008).

[29] I. Pastor, E. Vilaseca, S. Madurga, J. Garcés, M. Cascante, and F. Mas, J. Phys. Chem. B 114, 4028 (2010).

[30] E. Vilaseca, A. Isvoran, S. Madurga, I. Pastor, J. Garcés, and F. Mas, Phys. Chem. Chem. Phys. 13, 7396 (2011).

[31] E. Vilaseca, I. Pastor, A. Isvoran, S. Madurga, J.-L. Garcés, and F. Mas, Theor. Chem. Acc. 128, 795 (2011).

[32] P. C. Bressloff and J. M. Newby, Rev. Mod. Phys. 85, 135 (2013).

[33] S. P. Das, Statistical Physics of Liquids at Freezing and Beyond (Cambridge University Press, New York, 2011).

[34] J. A. Leegwater and G. Szamel, Phys. Rev. A 46, 4999 (1992).

[35] J. F. Brady, J. Fluid Mech. 272, 109 (1994).

[36] A. Imhof and J. K. G. Dhont, Phys. Rev. E 52, 6344 (1995).

[37] J. Happel and H. Brenner, Low Reynolds Number Hydrodynamics (Martinus Nijhoff, The Hague, 1983).

[38] M. Doi and S. F. Edwards, The Theory of Polymer Dynamics (Oxford University Press, Oxford, 1986).

[39] T. Miyaguchi, Phys. Rev. E 96, 042501 (2017).

[40] B. Felderhof, Phys. A (Amsterdam) 89, 373 (1977).

[41] J. Rotne and S. Prager, J. Chem. Phys. 50, 4831 (1969).

[42] H. Yamakawa, J. Chem. Phys. 53, 436 (1970).

[43] D. L. Ermak and J. A. McCammon, J. Chem. Phys. 69, 1352 (1978).

[44] R. R. Schmidt, J. G. H. Cifre, and J. G. de la Torre, J. Chem. Phys. 135, 084116 (2011).

[45] L. Durlofsky, J. F. Brady, and G. Bossis, J. Fluid Mech. 180, 21 (1987).

[46] J. F. Brady and G. Bossis, Annu. Rev. Fluid Mech. 20, 111 (1988).

[47] M. Tokuyama, T. Moriki, and Y. Kimura, Phys. Rev. E 83, 051402 (2011).

[48] P. M. Blanco, M. Via, J. L. Garcés, S. Madurga, and F. Mas, Entropy 19, 105 (2017).

[49] P. M. Blanco, J. L. Garcés, S. Madurga, and F. Mas, Soft Matter 14, 3105 (2018).

[50] M. Tokuyama and I. Oppenheim, Phys. Rev. E 50, R16 (1994).

[51] M. Tokuyama and I. Oppenheim, Phys. A (Amsterdam) 216, 85 (1995).

[52] M. Medina-Noyola, Phys. Rev. Lett. 60, 2705 (1988).
[53] R. P. Sear, J. Phys.: Condens. Matter 17, S3587 (2005).

[54] K. A. Dill, K. Ghosh, and J. D. Schmit, Proc. Natl. Acad. Sci. USA 108, 17876 (2011).

[55] A. van Blaaderen, J. Peetermans, G. Maret, and J. K. G. Dhont, J. Chem. Phys. 96, 4591 (1992).

[56] P. Atkins and J. De Paula, Atkins' Physical Chemistry (Oxford University Press, Oxford, 2018).

[57] R. Verberg, I. M. de Schepper, and E. G. D. Cohen, Phys. Rev. E 55, 3143 (1997).

[58] A. V. Weigel, B. Simon, M. M. Tamkun, and D. Krapf, Proc. Natl. Acad. Sci. USA 108, 6438 (2011).

[59] W. Deng and E. Barkai, Phys. Rev. E 79, 011112 (2009).

[60] Y. Meroz, I. M. Sokolov, and J. Klafter, Phys. Rev. E 81, 010101(R) (2010).

[61] T. Miyaguchi and T. Akimoto, Phys. Rev. E 91, 010102(R) (2015).

[62] M. V. Chubynsky and G. W. Slater, Phys. Rev. Lett. 113, 098302 (2014).

[63] T. Uneyama, T. Miyaguchi, and T. Akimoto, Phys. Rev. E 99, 032127 (2019).

[64] T. Miyaguchi, T. Uneyama, and T. Akimoto, Phys. Rev. E 100, 012116 (2019).

[65] M. Magdziarz, A. Weron, K. Burnecki, and J. Klafter, Phys. Rev. Lett. 103, 180602 (2009).

[66] M. Schwarzl, A. Godec, and R. Metzler, Sci. Rep. 7, 3878 (2017).

[67] P. E. Kloeden and E. Platen, Numerical Solution of Stochastic Differential Equations (Springer, Berlin, 2011).

[68] M. Matsumoto and T. Nishimura, ACM Trans. Model. Comput. Simul. 8, 3 (1998).

[69] B. J. Ackerson and L. Fleishman, J. Chem. Phys. 76, 2675 (1982).

[70] E. Yamamoto, T. Akimoto, M. Yasui, and K. Yasuoka, Sci. Rep. 4, 4720 (2014).

[71] E. Yamamoto, T. Akimoto, A. C. Kalli, K. Yasuoka, and M. S. P. Sansom, Sci. Adv. 3, e1601871 (2017).

[72] R. Metzler, J.-H. Jeon, A. G. Cherstvy, and E. Barkai, Phys. Chem. Chem. Phys. 16, 24128 (2014).

[73] T. Uneyama, T. Akimoto, and T. Miyaguchi, J. Chem. Phys. 137, 114903 (2012).

[74] T. Miyaguchi, T. Akimoto, and E. Yamamoto, Phys. Rev. E 94, 012109 (2016). 